\title{
Force-matched embedded-atom method potential for niobium
}

\author{
Michael R. Fellinger, ${ }^{*}$ Hyoungki Park, and John W. Wilkins \\ Department of Physics, The Ohio State University, Columbus, Ohio 43210, USA \\ (Received 4 January 2010; revised manuscript received 5 March 2010; published 19 April 2010)
}

\begin{abstract}
Large-scale simulations of plastic deformation and phase transformations in alloys require reliable classical interatomic potentials. We construct an embedded-atom method potential for niobium as the first step in alloy potential development. Optimization of the potential parameters to a well-converged set of density-functional theory (DFT) forces, energies, and stresses produces a reliable and transferable potential for moleculardynamics simulations. The potential accurately describes properties related to the fitting data and also produces excellent results for quantities outside the fitting range. Structural and elastic properties, defect energetics, and thermal behavior compare well with DFT results and experimental data, e.g., DFT surface energies are reproduced with less than $4 \%$ error, generalized stacking-fault energies differ from DFT values by less than $15 \%$, and the melting temperature is within $2 \%$ of the experimental value.
\end{abstract}

DOI: 10.1103/PhysRevB.81.144119 PACS number(s): 34.20.Cf, 62.20. $-\mathrm{x}, 65.40 .-\mathrm{b}, 61.72 . J-$

\section{INTRODUCTION}

Niobium's low-density, high melting temperature, and biocompatibility make it an attractive material for alloy design. $\mathrm{Nb}$ alloys are promising candidate materials for a wide variety of technological applications. Multifunctional Tibased "gum metal" alloys with substantial $\mathrm{Nb}$ concentrations exhibit remarkable properties and unique deformation behavior. ${ }^{1}$ Attempts to increase the operating temperatures, and hence efficiencies, of turbine engines have prompted interest in designing Nb-based superalloys. ${ }^{2}$ Nontoxic Ti-Nb based shape-memory alloys offer an alternative to Ti-Ni alloys for biomedical applications. ${ }^{3}$ Accurate and efficient computational models will aide in the microscopic understanding of deformation and transformation processes in these materials.

Advances in computational hardware and algorithms allow application of first-principles methods to increasingly complex problems. However, there remain calculations beyond the realm of $a b$ initio methods. Meaningful simulations of processes involving long-ranged strain fields or longwavelength fluctuations require large numbers of atoms. Therefore, methods must be developed that scale more favorably with system size than first-principles calculations while retaining a high degree of accuracy. The computational cost for simulations based on short-ranged classical potentials scales linearly with system size, allowing routine simulations of millions of atoms. However, the potentials must be carefully constructed and thoroughly tested to ensure that they yield reliable results. We construct a classical potential for large-scale $\mathrm{Nb}$ simulations and subsequent incorporation into potentials for alloys including Ti-Nb.

A number of authors have developed classical $\mathrm{Nb}$ potentials based on analytic functions. ${ }^{4-15}$ Analytic potentials are typically fit to experimental data for a small number of properties. These potentials reproduce the fit data with high accuracy but they often have limited transferability and can produce inaccurate forces for molecular-dynamics (MD) simulations. The force-matching method proposed by Ercolessi and Adams ${ }^{16}$ offers an alternative way to construct potentials. The functions are parameterized by cubic splines, and the spline knots are fit to a large number of forces from density-functional theory (DFT) (Refs. 17 and 18) calculations and usually experimental data as well. Including force data from different thermodynamic conditions improves accuracy and transferability for a larger range of simulations.

We use the force-matching method to develop a cubic spline-based embedded-atom method (EAM) potential ${ }^{19,20}$ for $\mathrm{Nb}$. The potential is fit to a database of DFT forces, energies, and stresses from $a b$ initio MD simulations. We do not fit to any experimental data since it may be inconsistent with the DFT information. Instead, we use experimental data and DFT results to test the accuracy of the potential. Section II discusses our DFT database calculations and potential optimization process. We utilize the force-matching program POTFIT (Refs. 21 and 22) to optimize the spline knots to the DFT database. Section III presents EAM calculations of structural and elastic properties, defects, and thermal behavior. We compare the results to DFT calculations and experimental data. These calculations demonstrate the potential's ability to describe properties related to the fitting data, as well as transferability to behavior beyond the fitting range.

\section{OPTIMIZATION OF THE EMBEDDED-ATOM METHOD POTENTIAL}

\section{A. Embedded-atom method interatomic potentials}

EAM potentials ${ }^{19,20}$ overcome limitations associated with simple pairwise interatomic potentials in simulations of metallic systems. Pair potentials yield a number of incorrect predictions for transition metals, ${ }^{23}$ including bond energies that are independent of the local bonding environment, a zero value for the Cauchy pressure $\left(C_{12}-C_{44}=0\right)$, and the equivalence of the cohesive energy with the unrelaxed vacancy formation energy. Supplementing the pairwise interaction with a volume-dependent term removes some of these undesirable features ${ }^{23,24}$ but the volume is ill defined near defects such as cracks and surfaces. EAM potentials overcome these difficulties by implicitly including many-body interactions and requiring the local "electronic density" as input rather than volume. 
The EAM formalism is based on ideas from DFT. ${ }^{17,18}$ The energy required to embed an impurity atom $Z$ in a solid at position $\mathbf{R}$ is a unique functional $\mathcal{F}_{Z, \mathbf{R}}[n]$ of the electronic density $n$ of the solid before the impurity is added. ${ }^{25,26}$ The embedded-atom method views each atom in the solid as an impurity embedded in a host solid made up of the remaining atoms. The energy functional is approximated by a potentialenergy function with two terms: (1) a sum of pairwise interactions $\phi_{s_{i} s_{j}}\left(\left|\mathbf{r}_{i}-\mathbf{r}_{j}\right|\right)$ between atoms $i$ and $j$, and (2) a sum of embedding energies $F_{s_{i}}\left(n_{i}\right)$ for each atom $i$ that depend on the local electronic density $n_{i}$ the atom sees from its surrounding neighbors. This local electronic density is a sum of radially symmetric electronic-density functions $\rho_{s_{j}}\left(\left|\mathbf{r}_{i}-\mathbf{r}_{j}\right|\right)$ arising from the atoms $j$ surrounding a given atom $i$,

$$
n_{i}=\sum_{j \neq i} \rho_{s_{j}}\left(r_{i j}\right),
$$

where $r_{i j}=\left|\mathbf{r}_{i}-\mathbf{r}_{j}\right|$ is the distance between atoms $i$ and $j$. The total potential energy is

$$
E=\sum_{i<j} \phi_{s_{i} s_{j}}\left(r_{i j}\right)+\sum_{i} F_{s_{i}}\left(n_{i}\right),
$$

where the subscripts $s_{i}$ and $s_{j}$ indicate that the functions depend on the species of the atoms.

Equations (1) and (2) are general and hold for multicomponent systems. The energy expression simplifies for monatomic systems,

$$
E=\sum_{i<j} \phi\left(r_{i j}\right)+\sum_{i} F\left(n_{i}\right),
$$

where

$$
n_{i}=\sum_{j \neq i} \rho\left(r_{i j}\right) .
$$

Thus, for a single component system the three functions $\phi$, $F$, and $\rho$ must be determined (whereas two-component alloys require seven functions). EAM potentials are implemented in many freely available MD codes, e.g., IMD, ${ }^{27,28}$ LAMMPS, ${ }^{29}$ and OHMMS. ${ }^{30}$ We perform our EAM calculations using all three of these codes.

\section{B. Database of DFT forces, energies, and stresses}

We use the force-matching method of Ercolessi and Adams $^{16}$ to obtain accurate potentials for moleculardynamics simulations. Force-matched potentials are fit to forces from DFT calculations and typically physical properties from either DFT calculations or experiment. Here we include only DFT data in our fitting database to avoid conflicting information. We use the force-matching program POTFIT (Refs. 21 and 22) to optimize the EAM functions to a database of DFT forces, energies per atom, and stresses for $\mathrm{Nb}$ from the configurations listed in Table I. Fitting to DFT data from configurations under different temperature and strain conditions extends the applicability of the potential to a wide range of simulations.

The DFT calculations are performed using the plane-wave program VASP. ${ }^{31}$ The Perdew-Burke-Ernzerhof (PBE) generalized-gradient approximation (GGA) functional ${ }^{32}$ accounts for the electronic exchange-correlation energy, and a projector augmented-wave (PAW) pseudopotential ${ }^{33}$ generated by $\mathrm{Kresse}^{34}$ represents the nucleus and core electrons. Along with the five valence states, the $4 s$ and $4 p$ semicore states are treated explicitly to accurately describe interactions at small interatomic separations. The elastic constants also agree better with experiment when more electronic states are included.

The database is calculated in two steps. First, $a b$ initio MD simulations generate realistic atomic trajectories for various temperature and strain conditions. The simulation supercells contain 124-128 atoms, depending on the structure. These calculations use a relatively low convergence criteria to reduce the computational burden. A single $k$ point is used, and the plane-wave cutoff energy is set to the default value of $219.927 \mathrm{eV}$ from the VASP pseudopotential file. Order-one Methfessel-Paxton smearing ${ }^{35}$ is used with a smearing width of $0.10 \mathrm{eV}$. The MD simulations run for 400 steps with a $3 \mathrm{fs}$ time step.

Second, well-converged calculations determine the forces, energies per atom, and stresses for the atomic configurations resulting from the final step of the MD simulations. We use $\Gamma$-centered $k$-point meshes with $30 \times 30 \times 30$ points per primitive cell and increase the plane-wave cutoff energy to $550 \mathrm{eV}$. The value of the smearing parameter is unchanged. The energies are converged to less than $1 \mathrm{meV} / \mathrm{atom}$. The fitting database contains 1895 forces (5685 force components), 15 energies per atom, and 90 stress tensor components from these calculations. Table I lists the configurations in the database, along with the weighted relative rms deviations of the EAM force magnitudes from the DFT values and the weighted average angular deviations of the EAM force directions from the DFT force directions (these errors are discussed in Sec. II E).

\section{Optimization of EAM functions to DFT data}

Generating accurate potentials using the force-matching method is an optimization problem in a high-dimensional space. The EAM functions are parameterized by cubic splines, and the program POTFIT (Refs. 21 and 22) optimizes the spline knots using a combination of simulated annealing and conjugate-gradientlike algorithms. Our potential construction procedure proceeds iteratively. We generate a database of DFT calculations and choose an initial set of spline knots. We also specify the cutoffs for the functions and the fitting weights for the values in the database. Then the optimization algorithms in POTFIT adjust the spline knots to minimize the weighted error between the database values and the corresponding values produced by the EAM potential. If the fitting errors are too large and the potential fails to produce satisfactory results for physical properties, we add or remove configurations from the database, change the fitting weights and cutoffs, and/or modify the number and initial values of the spline knots, and refit the potential. This optimization and testing process is repeated until we obtain accurate potentials.

In POTFIT, the fitting error is defined through a least- 
TABLE I. Configurations in the fitting database. The "structure" column lists the crystal structure of each configuration. The "primitive" and "conventional" labels indicate if the supercell is based on the one-atom primitive cell or the two-atom conventional cell. The liquid configuration starts as a bcc lattice and then melts during the $a b$ initio MD simulation. The " $N_{\text {atoms }}$ " column lists the number of atoms in each configuration. The " $T$ " column lists the temperatures of the $a b$ initio MD simulations used to generate the configurations. The " $V / V_{0}$ " column lists the ratio of the supercell volume to the zero-temperature, equilibrium DFT volume of the bcc, fcc, or hcp structure, respectively. For configuration $13, V_{0}$ is the equilibrium volume of the bcc structure. The "strain" column indicates the strain applied to the supercells, where $M$ denotes a volumeconserving monoclinic strain and $O$ denotes a volume-conserving orthorhombic strain. The "rms deviation" column lists the weighted relative rms deviations of the EAM force magnitudes from the DFT values. The " $\theta_{\text {avg }}$ " column lists the weighted average angular deviation of the EAM force directions from the DFT force directions. The weighted relative rms force-magnitude deviation and weighted average angular deviation of the forces for all the configurations are $25 \%$ and $15.1^{\circ}$, respectively.

\begin{tabular}{|c|c|c|c|c|c|c|c|}
\hline Configuration & Structure & $N_{\text {atoms }}$ & $\begin{array}{c}T \\
(\mathrm{~K})\end{array}$ & $V / V_{0}$ & Strain & $\begin{array}{c}\text { rms deviation } \\
(\%)\end{array}$ & $\begin{array}{c}\theta_{\text {avg }} \\
(\mathrm{deg})\end{array}$ \\
\hline 1 & bcc, primitive & 125 & 300 & 0.90 & None & 18 & 11.7 \\
\hline 2 & bcc, primitive & 125 & 300 & 1.00 & None & 20 & 12.3 \\
\hline 3 & bcc, primitive & 125 & 300 & 1.10 & None & 29 & 18.4 \\
\hline 4 & bcc, primitive, vacancy & 124 & 300 & 1.00 & None & 38 & 17.9 \\
\hline 5 & bcc, conventional & 128 & 300 & 1.00 & $2 \%, M$ & 20 & 14.3 \\
\hline 6 & bcc, conventional & 128 & 300 & 1.00 & $1 \%, M$ & 22 & 16.1 \\
\hline 7 & bcc, conventional & 128 & 300 & 1.00 & $-1 \%, M$ & 21 & 14.0 \\
\hline 8 & bcc, conventional & 128 & 300 & 1.00 & $-2 \%, M$ & 23 & 15.0 \\
\hline 9 & bcc, conventional & 128 & 300 & 1.00 & $2 \%, O$ & 23 & 16.0 \\
\hline 10 & bcc, conventional & 128 & 300 & 1.00 & $-2 \%, O$ & 21 & 15.9 \\
\hline 11 & bcc, primitive & 125 & 1200 & 1.00 & None & 21 & 13.2 \\
\hline 12 & bcc, primitive & 125 & 2200 & 1.00 & None & 19 & 12.4 \\
\hline 13 & liquid, primitive & 125 & 5000 & 1.00 & None & 21 & 11.9 \\
\hline 14 & fcc, primitive & 125 & 300 & 1.00 & None & 36 & 29.0 \\
\hline 15 & hcp, conventional & 128 & 300 & 1.00 & None & 51 & 37.4 \\
\hline
\end{tabular}

squares target function formed from the differences between the EAM and DFT values,

$$
\mathcal{Z}=\mathcal{Z}_{\mathrm{F}}+\mathcal{Z}_{\mathrm{C}}
$$

where

$$
\mathcal{Z}_{\mathrm{F}}=\sum_{i=1}^{N_{\mathrm{A}}} \sum_{j=1}^{3} W_{i} \frac{\left(F_{i, x_{j}}^{\mathrm{EAM}}-F_{i, x_{j}}^{\mathrm{DFT}}\right)^{2}}{\left(F_{i, x_{j}}^{\mathrm{DFT}}\right)^{2}+\epsilon_{i}}
$$

and

$$
\mathcal{Z}_{\mathrm{C}}=\sum_{i=1}^{N_{\mathrm{C}}} W_{i} \frac{\left(A_{i}^{\mathrm{EAM}}-A_{i}^{\mathrm{DFT}}\right)^{2}}{\left(A_{i}^{\mathrm{DFT}}\right)^{2}+\epsilon_{i}} .
$$

Equation (6) is the relative deviation of the EAM forces from the DFT forces, where $N_{\mathrm{A}}$ is the number of atoms in the fitting database, $F_{i, x_{j}}$ is the $x_{j}$ th component of the force on atom $i, W_{i}$ is the weight associated with each force, and $\epsilon_{i}$ is a small number that prevents overweighting of very small, inaccurate forces. Equation (7) is the relative deviation between the EAM energies per atom and stresses and the DFT values, where $N_{\mathrm{C}}$ is the number of energies per atom and stress tensor components, $A_{i}$ is an energy or stress value, $W_{i}$ is the associated weight, and $\epsilon_{i}$ is a small number that pre- vents overweighting of numerically small data. The optimal spline knots minimize $\mathcal{Z}$. See Brommer and Gähler ${ }^{21,22}$ for POTFIT details.

We determine if there are more parameters in the potential than the fitting database can support, i.e., overfitting, by calculating the errors for a testing database of DFT forces, energies per atom, and stresses for bcc, fcc, and hcp configurations not included in the fitting database. If the errors for the testing database are much larger than the errors for the fitting database, there are likely too many parameters specifying the EAM functions. ${ }^{22,36,37} \mathrm{We}$ also test the optimized potential's ability to predict physical properties (see Sec. III). If the potential fails to adequately describe the databases and desired properties, we add or remove configurations from the databases, modify the fitting weights and cutoffs, and/or change the number and initial values of the spline knots, and the optimization and testing process repeats.

Typical of simulated annealing methods, several hundred iterations of this procedure were required to find a small number of reasonable potentials. For the fitting database listed in Table I, we find that potentials with 15-20 spline knots for $\phi$ and $\rho$ and 5-10 spline knots for $F$, and a cutoff radius of $4.75 \AA$ for $\phi$ and $\rho$ produce the most physically reasonable results, while giving similar force-matching errors of $20-30 \%$ for the fitting and testing databases. The 

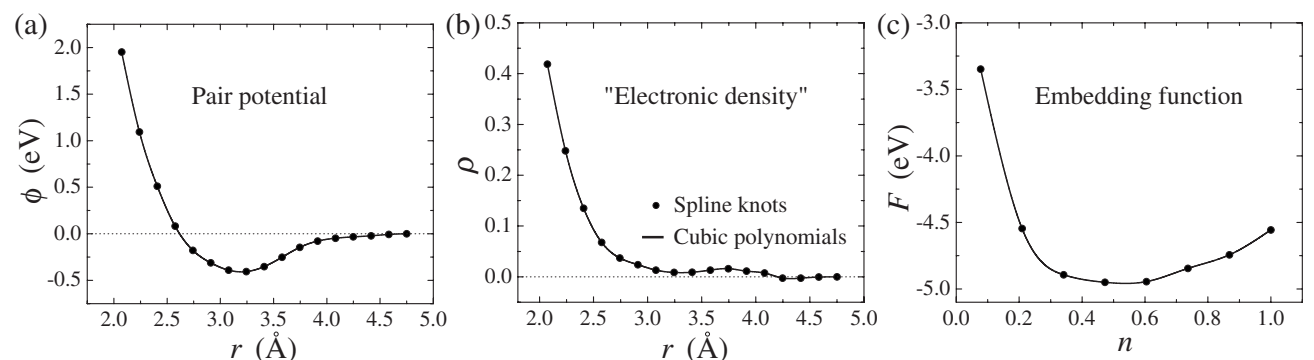

FIG. 1. The three cubic splines of the EAM potential. The points are optimized spline knots and the solid lines are cubic polynomials that interpolate between the knots. (a) The pair potential $\phi$ and (b) the electronic density $\rho$ are functions of the distance $r$ between pairs of atoms. Both of these functions have 17 optimized spline knots and a cutoff radius of $4.75 \AA$, which includes first-, second-, and third-nearestneighbor interactions in bcc $\mathrm{Nb}$. (c) The embedding function $F$ depends on the local electronic density $n$. Eight optimized spline knots parameterize $F$.

cutoff radius includes first-, second-, and third-nearestneighbor interactions in bcc $\mathrm{Nb}$. The cutoffs for $F$ are updated automatically by POTFIT as $\rho$ changes during optimization.

\section{Optimized potential}

Figure 1 shows the optimized cubic splines of the best $\mathrm{Nb}$ potential that we found. The pair potential $\phi$ and electronic density $\rho$ are parameterized using 17 equally spaced spline knots while eight spline knots are used for the embedding function $F$. The outer cutoff distance for $\phi$ and $\rho$ is $4.75 \AA$. The shortest interatomic distance in the fitting database, which is $2.073 \AA$, determines the inner cutoff distance. The inner and outer cutoffs of $F$ are 0.0775 and 1.000, respectively.

The function $\phi$ has the expected characteristics for a pair potential. The interaction is attractive for large interatomic separations and highly repulsive when atoms approach too closely. The minimum value for $\phi$ occurs at $r=3.197 \AA$. The electronic density $\rho$ is large for small interatomic separations and decreases for $r$ values up to about $3.25 \AA$. Beyond this distance, $\rho$ ripples and decays to zero at $4.75 \AA$. The zerotemperature equilibrium value of $n$ is 0.263 . We find that the nonmonotonic character of $\rho$ is required for an accurate description of $\mathrm{Nb}$. Potentials with smoother $\rho$ functions found during the optimization and testing procedure yield poor results for many properties. The embedding function $F$ has positive curvature over most of its range but there is a small region of negative curvature around $n=0.74$. This behavior is not ideal but atoms rarely sample $n$ values greater than 0.6 even at large temperatures and pressures.

In addition to specifying the spline knots and requiring continuity of the first and second derivatives of the functions at the knots, two boundary conditions must be applied to each function to determine all the cubic polynomial coefficients. The natural boundary condition, i.e., a vanishing second derivative, is applied at the inner cutoff radius of $\phi$ and $\rho$, and at the inner and outer cutoffs of $F$. The remaining boundary conditions are the first derivatives of $\phi$ and $\rho$ are zero at the outer cutoff radius. Appendix discusses modifications to $\phi, \rho$, and $F$ for small and large values of their arguments. These modifications improve the performance of the potential at large temperatures and pressures. Table II lists the spline knots and boundary conditions for $\phi, \rho$, and $F$. The potential functions are available in tabulated form upon request.

\section{E. Fitting errors}

The fitting database contains DFT forces, energies, and stresses for the configurations listed in Table I. The EAM potential computes the same set of quantities for the fixed atomic positions of each configuration and we evaluate the deviations of the EAM values from the corresponding DFT values. The errors associated with numerically small DFT data are typically much greater than the errors from larger data. This is illustrated in Figs. 2(a) and 2(b), which show the relative force-magnitude deviation and angular deviation of each of the EAM forces from the DFT database values, versus the DFT force magnitudes. Since very small values are inherently inaccurate, we weight the terms in the error calculations by the magnitudes of the DFT values. The weighted relative rms deviation of the energies per atom, stresses or force magnitudes is

$$
\Delta Q_{\mathrm{rms}}=\sqrt{\sum_{i=1}^{N_{\mathrm{Q}}} \omega_{i}\left(\frac{Q_{i}^{\mathrm{EAM}}-Q_{i}^{\mathrm{DFT}}}{Q_{i}^{\mathrm{DFT}}}\right)^{2}} \times 100 \%,
$$

where $Q_{i}$ is an energy per atom, a stress tensor component, or a force magnitude, and $N_{\mathrm{Q}}$ is the respective number of such quantities in the database. The scaled magnitudes of the DFT data $\omega_{i}$ weight the terms in the sum,

$$
\omega_{i}=\frac{\left|Q_{i}^{\mathrm{DFT}}\right|}{\sum_{j=1}^{N_{\mathrm{Q}}}\left|Q_{j}^{\mathrm{DFT}}\right|} .
$$

The EAM potential reproduces the energies per atom of the configurations with a weighted rms deviation of only $0.1 \%$. The diagonal components of the stress tensors are also accurately reproduced with a $6 \%$ weighted rms deviation. In contrast, the error for the off-diagonal components of the stress tensors is very large. The weighted rms deviation for these quantities is $307 \%$. The off-diagonal values are very small however, even for the strained supercells. Increasing the strain yields larger stress values but the DFT stress-strain 
TABLE II. The cubic spline knots and boundary conditions. Spline knots 1-17 for $\phi$ and $\rho$, and spline knots 1-8 for $F$ are optimized by POTFIT. The adjusted values of knot 0 for $\phi, \rho$, and $F$, and knot 9 for $F$ are also listed (see Appendix). The coefficients of the cubic polynomials that interpolate between the knots are determined by requiring continuity of the functions and their first and second derivatives, along with the boundary conditions.

\begin{tabular}{|c|c|c|c|c|c|}
\hline$i$ & $\begin{array}{c}r_{i} \\
(\AA)\end{array}$ & $\begin{array}{l}\phi\left(r_{i}\right) \\
(\mathrm{eV})\end{array}$ & $\rho\left(r_{i}\right)$ & $n_{i}$ & $\begin{array}{c}F\left(n_{i}\right) \\
(\mathrm{eV})\end{array}$ \\
\hline 0 & 1.7383750 & 5.644808063640994 & 0.683176019233847 & 0.000000000000000 & 0.000000000000000 \\
\hline 1 & 2.0730000 & 1.952032491449762 & 0.418661384304128 & 0.077492938439077 & -3.347285692522362 \\
\hline 2 & 2.2403125 & 1.094035979464646 & 0.248142385672424 & 0.209279661519209 & -4.546334492745762 \\
\hline 3 & 2.4076250 & 0.510885854762808 & 0.135151131573890 & 0.341066384599341 & -4.893456225397550 \\
\hline 4 & 2.5749375 & 0.082343335887366 & 0.067802030440920 & 0.472853107679473 & -4.950236437181159 \\
\hline 5 & 2.7422500 & -0.177550651790219 & 0.037078599738033 & 0.604639830759605 & -4.944970691786193 \\
\hline 6 & 2.9095625 & -0.311331931736446 & 0.023834158891363 & 0.736426553839736 & -4.845699482076931 \\
\hline 7 & 3.0768750 & -0.390004380615947 & 0.013226669087316 & 0.868213276919868 & -4.743717588952880 \\
\hline 8 & 3.2441875 & -0.405551151985570 & 0.008594239037838 & 1.000000000000000 & -4.556142211118433 \\
\hline 9 & 3.4115000 & -0.351882201216042 & 0.009026077313542 & 1.263573446160264 & 4.828348385154062 \\
\hline 10 & 3.5788125 & -0.251634925091355 & 0.013228711231271 & & \\
\hline 11 & 3.7461250 & -0.145378019920633 & 0.016102598867695 & & \\
\hline 12 & 3.9134375 & -0.078119761728408 & 0.011199412726043 & & \\
\hline 13 & 4.0807500 & -0.047220500113419 & 0.007407238328861 & & \\
\hline 14 & 4.2480625 & -0.032830828537903 & -0.002416625008422 & & \\
\hline 15 & 4.4153750 & -0.021236531023427 & -0.002572474995293 & & \\
\hline 16 & 4.5826875 & -0.006495370564318 & -0.000515878027624 & & \\
\hline 17 & 4.7500000 & 0.000000000000000 & 0.000000000000000 & & \\
\hline \multicolumn{6}{|c|}{ Boundary conditions } \\
\hline & $\phi^{\prime \prime}\left(r_{1}\right)=0$ & $\phi^{\prime}\left(r_{17}\right)=0$ & & & \\
\hline & $\rho^{\prime \prime}\left(r_{1}\right)=0$ & $\rho^{\prime}\left(r_{17}\right)=0$ & & & \\
\hline & $F^{\prime \prime}\left(n_{1}\right)=0$ & $F^{\prime \prime}\left(n_{8}\right)=0$ & & & \\
\hline
\end{tabular}

curves for $\mathrm{Nb}$ become nonlinear for strains larger than about $2.5 \%$. Table I lists the weighted relative rms deviations of the force magnitudes for each configuration in the database. The weighted relative rms deviation for all the configurations is
$25 \%$. This force-magnitude error is lower than the $32 \%$ error of Li et al.'s ${ }^{38}$ force-matched tantalum EAM potential and similar to the error of Hennig et al.'s ${ }^{39}$ force-matched titanium modified EAM (MEAM) potential. A direct compari-
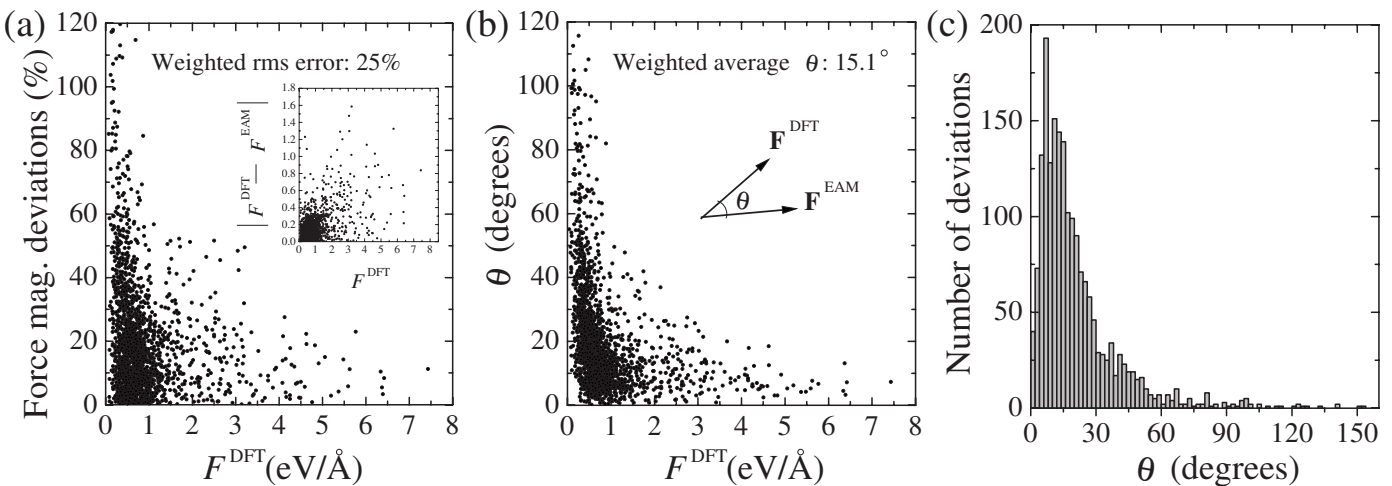

FIG. 2. Errors between the 1895 DFT forces in the fitting database and the corresponding EAM forces. The relative errors in the forces tend to decrease as the force magnitudes increase. (a) The percent relative deviation of the EAM force magnitudes from the DFT values, versus the magnitudes of the DFT forces. The weighted relative rms deviation of all the forces is $25 \%$. The inset shows the absolute errors in the forces. (b) The angular deviations of the EAM force directions from the corresponding DFT force directions. The weighted average of all the angular deviations is $15.1^{\circ}$. (c) Histogram of the angular deviations: $81 \%$ of the deviations are within the range $0<\theta<30^{\circ}, 96 \%$ of the deviations are within the range $0<\theta<60^{\circ}$, and $98 \%$ of the deviations are within the range $0<\theta<90^{\circ}$. 
son of the errors is difficult, however, due to the different types of potentials and/or configurations considered in each work.

We also determine the errors for the directions of the forces. The weighted average angular deviation of the EAM force directions from the DFT force directions is

$$
\theta_{\text {avg }}=\sum_{i=1}^{N_{\text {atoms }}} \omega_{i} \theta_{i},
$$

where $\theta_{i}$ is the angle between the EAM force on atom $i$ and the DFT force on atom $i$. Each angle in the sum is weighted by the corresponding scaled DFT force magnitude $\omega_{i}$. Table I lists the weighted average angular deviation of the forces for each database configuration. The weighted average angular deviation for all the configurations is only $15.1^{\circ}$. The histogram in Fig. 2(c) shows that $81 \%$ of the angular deviations are less than $30^{\circ}$ and $98 \%$ of the angular deviations are less than $90^{\circ}$.

The testing database contains 1381 forces from nine bcc configurations, one fcc configuration, and one hcp configuration. The data is generated for temperatures and pressures that lie between and beyond the temperatures and pressures in the fitting database. The weighted relative rms deviation of the EAM force magnitudes from the DFT values is $27 \%$, and the weighted average angular deviation of the EAM force directions from the DFT force directions is $16.5^{\circ}$. These values are very similar to the fitting database errors, indicating that the fitting database contains enough data to support the number of parameters in the potential.

\section{RESULTS AND DISCUSSION}

We assess the quality of the potential by comparing a wide variety of computed properties to DFT calculations and experimental data. All the DFT calculations use the same method and convergence criteria as the database calculations: PBE exchange-correlation functional, PAW pseudopotential with valence states and $4 s$ and $4 p$ semicore states treated explicitly, $\Gamma$-centered $k$-point meshes with $30 \times 30$ $\times 30$ points per primitive cell, a plane-wave cutoff energy of $550 \mathrm{eV}$, and order-one Methfessel-Paxton smearing with a smearing width of $0.10 \mathrm{eV}$. We calculate two classes of properties: (1) properties such as elastic constants which are directly related to configurations included in the fitting database and (2) properties such as surface energies which are not related to configurations included in the fitting database. The second class serves to test for overfitting and transferability. The potential performs well in nearly all situations we have tested.

\section{A. Structural and elastic properties}

The potential's first test is reproducing the cohesive energy, lattice parameter, and elastic properties of bcc $\mathrm{Nb}$. We also determine the energetic stability of the bcc lattice with respect to several other crystal structures. Table III compares the EAM results to our DFT calculations and experimental data. The cohesive energy, lattice parameter, and bulk modulus are determined by calculating the energy of bcc $\mathrm{Nb}$ for
TABLE III. The EAM values for the cohesive energy, lattice parameter, bulk modulus, and elastic constants of bcc Nb are compared to DFT and experiment. The experimental lattice parameter and elastic constants were measured at $4.2 \mathrm{~K}$. The EAM values for the energies and lattice parameters of the fcc, hcp, $\beta$-W, $\beta$-Ta, and $\omega$-Ti structures are compared to DFT results. The energies are relative to the energy of the bcc structure.

\begin{tabular}{|c|c|c|c|}
\hline & $\mathrm{EAM}^{\mathrm{a}}$ & GGA-PBE ${ }^{\mathrm{a}}$ & Experiment \\
\hline$E_{\mathrm{coh}}(\mathrm{eV} /$ atom $)$ & 7.09 & 7.10 & $7.57^{\mathrm{b}}$ \\
\hline$a(\AA)$ & 3.308 & 3.309 & $3.303^{\mathrm{c}}$ \\
\hline$B(\mathrm{GPa})$ & 172 & 172 & $173^{\mathrm{d}}$ \\
\hline$C_{11}(\mathrm{GPa})$ & 244 & 251 & $253^{\mathrm{d}}$ \\
\hline$C_{12}(\mathrm{GPa})$ & 136 & 133 & $133^{\mathrm{d}}$ \\
\hline$C_{44}(\mathrm{GPa})$ & 32 & 22 & $31^{\mathrm{d}}$ \\
\hline$\Delta E_{\mathrm{fcc}-\mathrm{bcc}}(\mathrm{meV} / \mathrm{atom})$ & 187 & 324 & $\cdots$ \\
\hline$a_{\mathrm{fcc}}(\AA)$ & 4.157 & 4.217 & $\ldots$ \\
\hline$\Delta E_{\mathrm{hcp}-\mathrm{bcc}}(\mathrm{meV} / \mathrm{atom})$ & 187 & 297 & $\cdots$ \\
\hline$a_{\mathrm{hcp}}(\AA)$ & 2.940 & 2.867 & $\cdots$ \\
\hline$c_{\text {hcp }}(\AA)$ & 4.800 & 5.238 & $\cdots$ \\
\hline$\Delta E_{\beta \mathrm{W}-\mathrm{bcc}}(\mathrm{meV} /$ atom $)$ & 77 & 104 & $\cdots$ \\
\hline$a_{\beta \mathrm{W}}(\AA)$ & 5.280 & 5.296 & $\cdots$ \\
\hline$\Delta E_{\beta \text { Ta-bcc }}(\mathrm{meV} /$ atom $)$ & 105 & 83 & $\cdots$ \\
\hline$a_{\beta \mathrm{Ta}}(\AA)$ & 10.200 & 10.184 & $\cdots$ \\
\hline$c_{\beta \mathrm{Ta}}(\AA)$ & 5.313 & 5.371 & $\cdots$ \\
\hline$\Delta E_{\omega \mathrm{Ti} \text {-bcc }}(\mathrm{meV} /$ atom $)$ & 167 & 201 & $\cdots$ \\
\hline$a_{\omega \mathrm{Ti}}(\AA)$ & 4.845 & 4.887 & $\cdots$ \\
\hline$c_{\omega \mathrm{Ti}}(\AA)$ & 2.735 & 2.678 & $\cdots$ \\
\hline
\end{tabular}

aThis work.

${ }^{\mathrm{b}}$ Experimental data from Kittel (Ref. 43).

${ }^{\mathrm{c}}$ Experimental data from Roberge (Ref. 44).

${ }^{\mathrm{d}}$ Experimental data from Simmons and Wang (Ref. 45). The bulk modulus is obtained from $C_{11}$ and $C_{12}: B=\left(C_{11}+2 C_{12}\right) / 3$.

the volume range $0.90 V_{0}<V<1.10 V_{0}$, where $V_{0}$ is the equilibrium volume, and fitting the third-order Birch-Murnaghan equation of state ${ }^{40-42}$ to the results. DFT produces a cohesive energy $6 \%$ lower than the experimental value. ${ }^{43}$ The EAM cohesive energy is slightly different than the DFT value since the DFT energies per atom of several structures under different thermodynamic conditions are used to construct the potential rather than the zero-temperature energy per atom. Both DFT and the EAM potential reproduce the lattice parameter measured at $4.2 \mathrm{~K}$ (Ref. 44) with an error of less than $1 \%$. The DFT and EAM bulk modulus values closely match the experimental result, ${ }^{45}$ each with an error of less than $1 \%$. The bulk modulus for cubic crystals is related to the elastic constants $C_{11}$ and $C_{12}$ via $B=\left(C_{11}+2 C_{12}\right) / 3$.

We compute the elastic constants $C^{\prime}=\left(C_{11}-C_{12}\right) / 2$ and $C_{44}$ by straining the bcc crystal and calculating the resulting stress. The slopes of the stress versus stain curves yield the elastic constants. We use a volume-conserving orthorhombic strain to compute $C^{\prime}$ and a volume-conserving monoclinic strain for $C_{44}{ }^{41}$ We apply a range of strains from $-1 \%$ to $+1 \%$ in each case. $B$ and $C^{\prime}$ determine $C_{11}$ and $C_{12}$. The errors of the EAM elastic constants compared to 
TABLE IV. Single-vacancy formation, migration, and diffusion activation energies. The activation energy is the sum of the formation and migration energies: $Q_{\mathrm{vac}}=E_{\mathrm{vac}}^{\mathrm{f}}+E_{\mathrm{vac}}^{\mathrm{m}}$. Energies are reported in $\mathrm{eV}$.

\begin{tabular}{lcccccccc}
\hline \hline & EAM $^{\mathrm{a}}$ & GGA-PBE $^{\mathrm{a}}$ & Experiment & EAM $^{\mathrm{b}}$ & EAM $^{\mathrm{c}}$ & F-S $^{\mathrm{d}}$ & MEAM $^{\mathrm{e}}$ & MEAM $^{\mathrm{f}}$ \\
\hline$E_{\text {vac }}^{\mathrm{f}}$ & 3.10 & 2.72 & $2.6-3.1^{\mathrm{g}}$ & 2.88 & 2.76 & 2.48 & 2.75 & 2.75 \\
$E_{\text {vac }}^{\mathrm{m}}$ & 0.77 & 0.55 & $0.6-1.6^{\mathrm{g}}$ & 0.97 & 0.64 & 0.91 & 0.54 & 0.57 \\
$Q_{\text {vac }}$ & 3.87 & 3.27 & $3.6-4.1^{\mathrm{h}}$ & 3.85 & 3.40 & 3.39 & 3.29 & 3.32 \\
\hline \hline
\end{tabular}

\footnotetext{
This work.

${ }^{b}$ EAM results of Guellil and Adams (Ref. 8).

${ }^{\mathrm{C}} \mathrm{EAM}$ results of $\mathrm{Hu}$ et al. (Ref. 12).

${ }^{\mathrm{d}} \mathrm{F}-\mathrm{S}$ results of Harder and Bacon (Ref. 51).

'MEAM results of Zhang et al. (Ref. 10).

${ }_{\mathrm{f}} \mathrm{MEAM}$ results of Lee et al. (Ref. 11).

${ }^{\mathrm{g}}$ Experimental data from Landolt-Börnstein (Ref. 52).

${ }^{\mathrm{h}}$ Experimental data from Refs. 52-56.
}

experiment $^{45}$ are $4 \%, 2 \%$, and $3 \%$ for $C_{11}, C_{12}$, and $C_{44}$, respectively. The measured values are from single crystals at 4.2 K. In principle, the EAM value for $C_{44}$ should closely match the DFT value since no experimental data is used to fit the potential. The POTFIT program fits to DFT stresses rather than the elastic constants and the off-diagonal stress tensor component $\sigma_{x y}$ determines $C_{44}$. The off-diagonal stress tensor components in the fitting database are generally much smaller than the diagonal components, and it is difficult to fit potentials that yield accurate $C_{44}$ values. For example, the largest $\sigma_{x x}$ value in the database is $22.4 \mathrm{GPa}$ while the largest $\sigma_{x y}$ value is only $0.490 \mathrm{GPa}$. A large stress fitting weight must be used to produce potentials with $C_{44}$ values close to the DFT and experimental values.

The stability of the bcc crystal structure is demonstrated with respect to the fcc and hep structures. DFT predicts that the energies per atom for fcc and hcp $\mathrm{Nb}$ are $323 \mathrm{meV}$ and 296 meV larger than the bcc value, respectively. The EAM potential predicts that the energy per atom for both of these structures is $187 \mathrm{meV}$ larger than the bcc value. Our EAM potential produces the ideal close-packed $c / a$ value of 1.633 for the hcp structure, whereas the DFT value is $c / a=1.827$. For $c / a=1.633$, the fcc and hcp first-nearest-neighbor distances are equal, as are the second-nearest-neighbor distances. Therefore, third-nearest-neighbor interactions must be included to differentiate between fcc and hcp for potentials with no angular dependence. Our EAM potential includes first-, second-, and third-nearest-neighbor interactions in the bcc structure, but only first- and second-nearestneighbor interactions for the fcc and hcp structures. This leads to the ideal $c$ / $a$ ratio in the hcp structure and energetic degeneracy of the fcc and hcp structures. Increasing the range of $\phi$ and $\rho$ and including more fcc and hep data in the fitting database produces values closer to the DFT results, but the bcc elastic constants, phonon dispersions, and vacancy formation energy agreed poorly with DFT and experiment.

The bcc metals tungsten (W) and tantalum (Ta) have metastable $\beta$ phases based on structures with eight atoms per unit cell and 30 atoms per unit cell, respectively. The $\beta-\mathrm{W}$ structure has $P m \overline{3} n$ symmetry and the $\beta$-Ta structure has $\mathrm{P}_{2} /$ mnm symmetry. Titanium (Ti) transforms from hep to bcc at $1155 \mathrm{~K}$ and ambient pressure, and has a high-pressure $\omega$ phase based on a three-atom unit cell with $P 6 / \mathrm{mmm}$ symmetry. No data for the $\beta-\mathrm{W}, \beta$-Ta, and $\omega$-Ti structures is included in the fitting database. The energies of these structures are higher than the bcc energy. The lattice parameters of all the structures are reproduced reasonably well with the largest error for the hcp $c$ value. The energetic ordering of the structures is different in EAM and DFT but bcc is most stable in both cases. We also find that in finite-temperature MD simulations, the EAM potential stabilizes the bcc structure to the melting point for pressures below $125 \mathrm{GPa}$ (see Sec. III C).

\section{B. Point defects}

Vacancy motion is the predominant mechanism for solidstate diffusion and the presence of vacancies influences many material processes including dislocation motion and creep. We use our EAM potential to calculate the single-vacancy formation energy $E_{\mathrm{vac}}^{\mathrm{f}}$ and migration energy $E_{\mathrm{vac}}^{\mathrm{m}}$, and the activation energy for vacancy diffusion $Q_{\mathrm{vac}}=E_{\mathrm{vac}}^{\mathrm{f}}+E_{\mathrm{vac}}^{\mathrm{m}}$. The simulation supercells contain 8191 atoms. We determine the vacancy migration energy with the nudged elastic-band method $^{46-48}$ using seven image configurations between the initial and final configurations. The migration path is along the $\langle 111\rangle$ direction. We also compute the vacancy energies using DFT for supercells with 249 atoms. In all our calculations, the atoms are relaxed using the conjugate-gradient method. 49,50

Table IV compares our EAM vacancy energies to our DFT results and other published EAM, ${ }^{8,12}$ Finnis-Sinclair (F-S), ${ }^{51}$ and MEAM (Ref. 11) calculations. Most of the results are consistent with the experimental data. ${ }^{52-56}$ Our EAM potential produces the largest formation energy. The POTFIT program fits to the DFT energy per atom of each configuration in the database, instead of fitting to defect energies. The DFT energy difference per atom between an ideal crystal and a crystal with a single vacancy is about $10 \mathrm{meV}$. This is close to the accuracy with which the EAM potential reproduces the energies in the fitting database and a large energy fitting weight must be used to achieve reasonable results. 


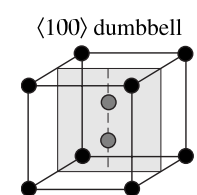

(a)

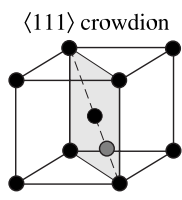

(d)

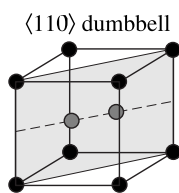

(b)

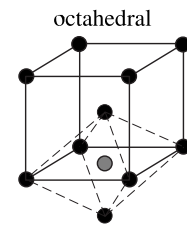

(e)

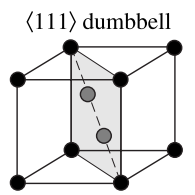

(c)

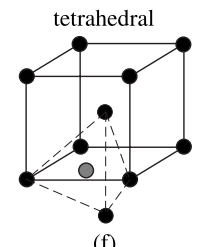

(f)
FIG. 3. Schematic illustration of the (a) $\langle 100\rangle$ dumbbell, (b) $\langle 110\rangle$ dumbbell, (c) $\langle 111\rangle$ dumbbell, (d) $\langle 111\rangle$ crowdion, (e) octahedral, and (f) tetrahedral interstitials.

In the absence of strong irradiation, the equilibrium concentration of self-interstitial atoms in metals is much smaller than the concentration of vacancies. Accordingly, no data from configurations with interstitials is included in the fitting database. Instead, interstitial formation energy calculations can test the transferability of the EAM potential. We determine the formation energies of six self-interstitial configurations: the $\langle 100\rangle$ dumbbell, $\langle 110\rangle$ dumbbell, $\langle 111\rangle$ dumbbell, $\langle 111\rangle$ crowdion, octahedral, and tetrahedral interstitials. Figure 3 shows the geometry of these defects. The EAM simulation supercells contain 31251 atoms which are relaxed using the conjugate-gradient method. Since no experimental data is available, we also compute the formation energies with DFT. The DFT supercells contain 251 atoms which are relaxed with the conjugate-gradient method.

Table V lists our EAM results, along with our DFT values and other published EAM, ${ }^{12}$ F-S, ${ }^{5,6,57}$ and MEAM (Ref. 11) results. Our EAM potential yields self-interstitial formation energies in the range 3.83-4.50 eV and our DFT calculations give formation energies in the range 3.95-4.89 eV. DFT predicts that the $\langle 111\rangle$ dumbbell has the lowest energy while our EAM potential predicts the $\langle 110\rangle$ dumbbell to be the lowest. The EAM results of $\mathrm{Hu}$ et al. ${ }^{12}$ and the F-S results of Ack- land and Thetford, ${ }^{5}$ Rebonato et al. ${ }^{6}$ and Harder and Bacon ${ }^{57}$ also place the formation energy of the $\langle 110\rangle$ dumbbell lowest. Since our EAM results are not consistent with DFT, the potential may not be well suited for radiation damage studies.

\section{Phonon-dispersion, thermal-expansion, and pressure-volume relation}

The next group of properties relate to lattice vibrations and the thermodynamic behavior of the potential. The calculations demonstrate the applicability of the potential over a large range of temperatures and pressures. First, we use the program PHON (Ref. 58) to compute the phonon spectra along high-symmetry directions in the Brillouin zone. The program employs the small-displacement method, in which atoms are moved a small distance from their equilibrium lattice sites. The dynamical matrix obtained from the resulting forces on the atoms yields the phonon dispersions.

Figure 4 compares the computed phonon spectra along the $[\xi 00],[\xi \xi \xi]$, and $[\xi \xi 0]$ directions in reciprocal space to experimental data, ${ }^{59}$ our DFT calculations, and other published EAM results. ${ }^{8,12}$ The DFT calculations are carried out for up to 512 atoms $(8 \times 8 \times 8$ supercells $)$. The DFT results closely match the experimental data over much of the Brillouin zone but the transverse modes in the $[\xi 00]$ direction show a plateau around $\xi=0.25$ which is not present in the experimental data. This discrepancy is not physical but rather is an artifact of the interpolation scheme used in generating the curves. The phonon frequencies are computed exactly at only a small number of points in the Brillouin zone and PHON interpolates between these exact values to generate smooth curves. More exact points, i.e., even larger supercells, are required to remove this discrepancy. Our EAM potential accurately describes the experimental phonon frequencies for small wave vectors but is unable to reproduce some of the features in the spectrum. This results in poor agreement at the zone boundaries $\mathrm{H}$ and $\mathrm{N}$ but our EAM results match experiment more closely than the EAM results of Guellil and Adams ${ }^{8}$ and $\mathrm{Hu}$ et al. ${ }^{12}$

TABLE V. Formation energies for the $\langle 100\rangle$ dumbbell, $\langle 110\rangle$ dumbbell, $\langle 111\rangle$ dumbbell, $\langle 111\rangle$ crowdion, octahedral, and tetrahedral interstitials in electron volt. The lowest formation energy for each calculation is underlined. No DFT interstitial data is used in constructing our EAM potential.

\begin{tabular}{lccccccc}
\hline \hline & EAM $^{\mathrm{a}}$ & GGA-PBE $^{\mathrm{a}}$ & EAM $^{\mathrm{b}}$ & F-S $^{\mathrm{c}}$ & F-S $^{\mathrm{d}}$ & F-S $^{\mathrm{e}}$ & MEAM $^{\mathrm{f}}$ \\
\hline$E_{100}^{\mathrm{f}}$ & 4.50 & 4.76 & 4.44 & 4.13 & 4.821 & 4.85 & $\ldots$ \\
$E_{110}^{\mathrm{f}}$ & 3.83 & 4.31 & 4.39 & 3.99 & 4.485 & 4.54 & 2.56 \\
$E_{111}^{\mathrm{f}}$ & 4.09 & 3.95 & 4.74 & $\ldots$ & 4.795 & 4.88 & $\ldots$ \\
$E_{\text {crd }}^{\mathrm{f}}$ & 4.02 & 3.99 & 4.93 & 4.10 & 4.857 & 4.95 & $\ldots$ \\
$E_{\text {oct }}^{\mathrm{f}}$ & 4.36 & 4.89 & 4.43 & 4.23 & $\ldots$ & 4.91 & $\ldots$ \\
$E_{\text {tet }}^{\mathrm{f}}$ & 4.37 & 4.56 & 4.73 & 4.26 & $\ldots$ & 4.95 & $\cdots$ \\
\hline \hline
\end{tabular}

This work.

${ }^{b}$ EAM results of Hu et al. (Ref. 12).

${ }^{\mathrm{c}} \mathrm{F}-\mathrm{S}$ results of Rebonato et al. (Ref. 6).

${ }^{\mathrm{d}} \mathrm{F}-\mathrm{S}$ results of Ackland and Thetford (Ref. 5).

${ }^{\mathrm{e}} \mathrm{F}-\mathrm{S}$ results of Harder and Bacon (Ref. 57).

${ }^{\mathrm{f}}$ MEAM result of Lee et al. (Ref. 11). 


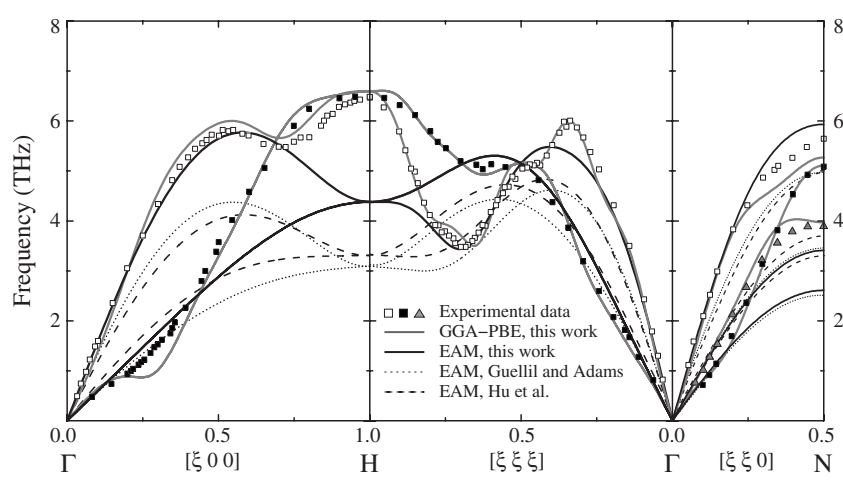

FIG. 4. Phonon-dispersion curves along high-symmetry directions. Our EAM results agree well with experiment (Ref. 59) for small wave vectors but the classical EAM potential is unable to fully capture the spectrum. However, the potential improves upon the EAM results of Guellil and Adams (Ref. 8) and Hu et al. (Ref. 12). The DFT phonon results closely match the experimental data over much of the Brillouin zone. The transverse modes in the [ $\xi 00]$ direction show a plateau around $\xi=0.25$ which is an artifact of the interpolation scheme used to generate the curves. The DFT phonon results were provided by Hennig.

Figure 5(a) shows the thermal expansion of the EAM potential from $0 \mathrm{~K}$ to the experimental melting temperature, $T_{\text {melt }}^{\text {exp }}=2742 \mathrm{~K}$. Constant $-N P T$ MD simulations of 8192 atoms at $P=1$ atm yield the thermal expansion curve. We de-
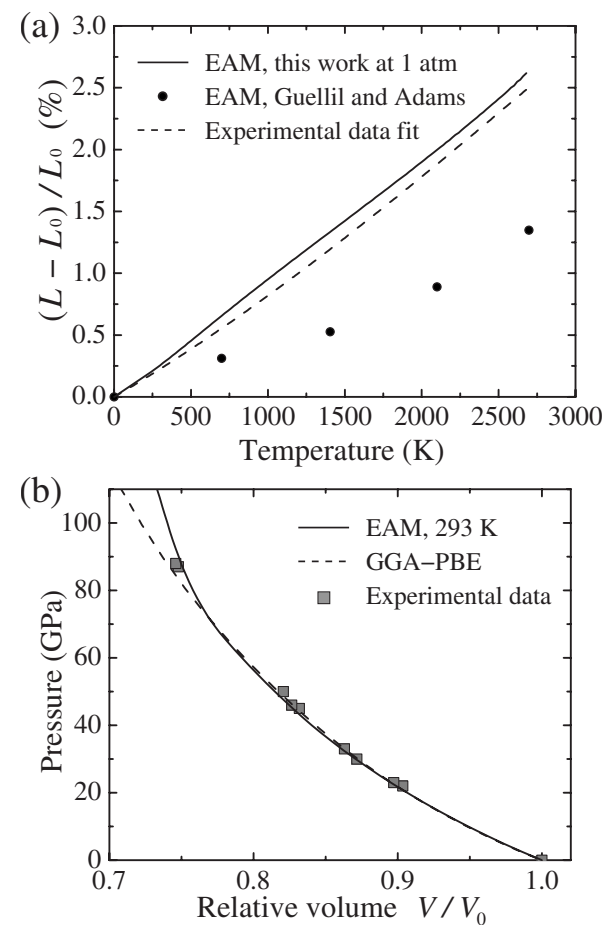

FIG. 5. (a) Thermal expansion curve. The thermal expansion of our EAM potential agrees closely with experiment (Ref. 60) from 0 $\mathrm{K}$ to $T_{\text {melt }}^{\exp }=2742 \mathrm{~K}$. Our EAM potential slightly overestimates the thermal expansion while the EAM potential of Guellil and Adams (Ref. 8) underestimates it. (b) Pressure versus volume curve. The experimental data are from shock experiments (Ref. 61). For pressures to $75 \mathrm{GPa}$, our EAM potential agrees well with experimental data and our DFT calculations. termine the equilibrium lattice constant for 138 temperatures in the range $0<T<2742 \mathrm{~K}$. Each $\mathrm{MD}$ simulation runs for 500000 steps with a 1 fs time step and we determine the lattice constant for each temperature by averaging over the last 5000 simulation steps. We compare the results to experimental data ${ }^{60}$ and the EAM results of Guellil and Adams. ${ }^{8}$ Our EAM result lies just above the experimental curve while the Guellil and Adams potential underestimates the expansion. Our fitting database contains data for bcc Nb only at (i) $300 \mathrm{~K}$ and -13 to $23 \mathrm{GPa}$, (ii) $1200 \mathrm{~K}$ and $2 \mathrm{GPa}$, and (iii) $2200 \mathrm{~K}$ and $7 \mathrm{GPa}$, so our results indicate the potential accurately interpolates to temperatures and pressures not included in the fit.

Figure 5(b) shows the pressure variation in the EAM potential versus the relative volume $V / V_{0}$, where $V_{0}$ is the zeropressure volume. Constant-NPT MD simulations of 8192 atoms at $T=293 \mathrm{~K}$ yield the pressure-volume curve. We determine the equilibrium volume for 50 pressures in the range $0<P<125 \mathrm{GPa}$. Each $\mathrm{MD}$ simulation runs for 500000 steps with a 1 fs time step, and we determine the volume for each pressure by averaging over the last 5000 simulation steps. Zero-temperature EAM results are nearly identical to the $293 \mathrm{~K}$ values. We compare the results to data from shock experiments ${ }^{61}$ and our zero-temperature DFT calculations. For pressures to $75 \mathrm{GPa}$ the agreement with DFT and experiment is excellent. The largest pressure in the fitting database is only $23 \mathrm{GPa}$ from configuration 1 in Table I, and the compression of bcc $\mathrm{Nb}$ is accurately reproduced for more than $50 \mathrm{GPa}$ beyond this pressure. The EAM result deviates at larger pressures and at $125 \mathrm{GPa}$ the bcc crystal structure transforms to a close-packed lattice. Experiment and DFT do not show a phase transformation. Therefore the potential may not be well suited for shock simulations but it performs very well below $75 \mathrm{GPa}$.

\section{Surface properties}

Surface properties test the transferability of our potential to configurations with low coordination number since no surface data is used in constructing the potential. Table VI lists the relaxed surface energies for the $\{110\},\{100\}$, and $\{111\}$ surfaces. The EAM calculations use slab-geometry supercells with two free surfaces and periodic boundary conditions in the directions perpendicular to the surface normals. The conjugate-gradient method relaxes 600-layer slabs in directions parallel to the surface normals while the perpendicular dimensions are fixed. We compare the surface energies to our DFT results and published EAM, ${ }^{8,12} \mathrm{~F}-\mathrm{S},{ }^{62}$ long-range empirical potential (LREP), ${ }^{13}$ MEAM,,${ }^{9,11}$ and modified analytical EAM (MAEAM) (Ref. 14) results. Experimental values for energies of individual surfaces are often based on simple models, so we evaluate the accuracy of the EAM surface energies by comparing the results to our DFT calculations.

We perform DFT calculation for 24-, 36-, and 48-layer slabs for the $\{100\}$ surface, and for 12-, 18-, and 24-layer slabs for the $\{110\}$ and $\{111\}$ surfaces. A vacuum region $10 \AA$ thick separates the periodic surface images. We relax the slabs in a manner similar to the EAM calculations. We 
TABLE VI. Low-index surface energies of bcc $\mathrm{Nb}$ in $\mathrm{meV} / \AA^{2}$ $\left(\mathrm{J} / \mathrm{m}^{2}\right)$. Our EAM results closely match our DFT values, even though no surface data is used to construct the potential.

\begin{tabular}{lccc}
\hline \hline & $E_{\text {surf }}^{\{110\}}$ & $E_{\text {surf }}^{\{100\}}$ & $E_{\text {surf }}^{\{111\}}$ \\
\hline EAM $^{\mathrm{a}}$ & $127(2.04)$ & $147(2.36)$ & $154(2.47)$ \\
GGA-PBE $^{\mathrm{a}}$ & $131(2.10)$ & $146(2.34)$ & $149(2.39)$ \\
EAM $^{\mathrm{b}}$ & $113(1.81)$ & $123(1.97)$ & $\ldots$ \\
EAM $^{\mathrm{c}}$ & $108(1.73)$ & $120(1.93)$ & $\ldots$ \\
F-S $^{\mathrm{d}}$ & $104(1.67)$ & $122(1.96)$ & $\ldots$ \\
LREP $^{\mathrm{e}}$ & $112(1.79)$ & $131(2.10)$ & $146(2.34)$ \\
MEAM $^{\mathrm{f}}$ & $117(1.87)$ & $174(2.79)$ & $126(2.02)$ \\
MEAM & $155(2.49)$ & $169(2.72)$ & $182(2.92)$ \\
MAEAM & $110(1.77)$ & $125(2.00)$ & $143(2.28)$ \\
\hline \hline
\end{tabular}

aThis work.

${ }^{\mathrm{b}}$ EAM results of Guellil and Adams (Ref. 8).

${ }^{c}$ EAM results of Hu et al. (Ref. 12).

${ }^{\mathrm{d}} \mathrm{F}-\mathrm{S}$ results of Ackland and Finnis (Ref. 62).

${ }^{\text {}}$ LREP results of Dai et al. (Ref. 13). Unrelaxed surface energies. ${ }^{f}$ MEAM results of Baskes (Ref. 9). Unrelaxed surface energies.

gMEAM results of Lee et al. (Ref. 11).

${ }^{h}$ MAEAM results of Wen and Zhang (Ref. 14).

use different numbers of layers to study the convergence of the surface energies and relaxations with cell size. The energy values for the different numbers of layers vary by $1 \mathrm{meV} / \AA^{2}$ or less. When we increase the vacuum layer thickness to $15 \AA$ for the largest supercells, the surface energies change by less than $0.2 \mathrm{meV} / \AA^{2}$. The errors between our EAM and DFT results for the $\{110\},\{100\}$, and $\{111\}$ surfaces are $3.1 \%, 0.7 \%$, and $3.4 \%$, respectively. Both methods predict that $E_{\text {surf }}^{\{110\}}<E_{\text {surf }}^{\{100\}}<E_{\text {surf }}^{\{111\}}$. The excellent agreement between our EAM and DFT results is surprising, considering the fitting database does not contain configurations with surfaces. All the potentials give reasonable surface energies with respect to the DFT results. The relative rms deviation of our EAM values from the DFT values is $2.6 \%$. Dai et al.' ${ }^{13}$ LREP potential has the next lowest relative rms deviation of $10.3 \%$, and Lee et al.'s ${ }^{11}$ MEAM potential has the highest relative rms deviation of $18.9 \%$. Baskes' ${ }^{9}$ unrelaxed MEAM results show a different ordering of the energies than DFT.

Table VII lists the percent change in spacing between the first and second surface layers relative to the spacing in the bulk. We compare our EAM results to our DFT calculations, experimental data, ${ }^{63}$ and published $\mathrm{EAM}^{8}{ }^{8} \mathrm{~F}-\mathrm{S},{ }^{62}$ and MEAM (Ref. 11) results. Our EAM values agree very closely with our DFT calculations. All the methods produce contractions of the $\{110\},\{100\}$, and $\{111\}$ surface layers, except the EAM potential of Guellil and Adams which predicts an expansion of the $\{100\}$ layers. Our $\{100\}$ EAM and DFT results also agree well with experiment. We do not list relaxations for layers deeper beneath the surface since the DFT results oscillate strongly as the number of layers in the slab changes.

\section{E. Stacking faults and dislocations}

The nonplanar core of screw dislocations in bcc transition metals is generally accepted to be responsible for the com-
TABLE VII. Low-index surface relaxations of bcc $\mathrm{Nb}$. The values are the relative percent change in the interplanar spacing upon relaxation. The number of layers in our slabs are in parentheses.

\begin{tabular}{lccc}
\hline \hline & $\begin{array}{c}\Delta_{12}^{\{110\}} \\
(\%)\end{array}$ & $\begin{array}{c}\Delta_{12}^{\{100\}} \\
(\%)\end{array}$ & $\begin{array}{c}\Delta_{12}^{\{111\}} \\
(\%)\end{array}$ \\
\hline EAM $^{\mathrm{a}}$ & $-5.0(600)$ & $-13.9(600)$ & $-27.0(600)$ \\
GGA-PBE $^{\mathrm{a}}$ & $-3.9(12)$ & $-12.4(24)$ & $-30.7(12)$ \\
GGA-PBE $^{\mathrm{a}}$ & $-3.9(18)$ & $-13.0(36)$ & $-28.4(18)$ \\
GGA-PBE $^{\mathrm{a}}$ & $-4.5(24)$ & $-12.3(48)$ & $-27.6(24)$ \\
Experiment $^{\mathrm{b}}$ & $\ldots$ & $-13 \pm 5$ & $\ldots$ \\
EAM $^{\mathrm{c}}$ & -1.6 & +0.52 & $\ldots$ \\
F-S $^{\mathrm{d}}$ & -5.1 & -16.0 & $\ldots$ \\
MEAM $^{\mathrm{e}}$ & -7.3 & -12.5 & -35.5 \\
\hline \hline
\end{tabular}

This work.

${ }^{\text {b}}$ Experimental data from Lo et al. (Ref. 63).

${ }^{\mathrm{c}}$ EAM results of Guellil and Adams (Ref. 8).

${ }^{\mathrm{d}}$ F-S results of Ackland and Finnis (Ref. 62).

${ }^{\mathrm{e} M E A M}$ results of Lee et al. (Ref. 11).

plex deformation behavior of these materials. ${ }^{64-71}$ The cores of $(1 / 2)\langle 111\rangle$ screw dislocations in bcc metals spread into several planes of the $\langle 111\rangle$ zone. However, no dissociation of dislocations into well-defined partial dislocations has been observed, and no metastable stacking faults that could participate in such dissociation have been identified. The most widely used theoretical approach in searching for possible stacking faults is $\gamma$-surface calculations. The $\gamma$ surfaces represent energies of generalized stacking faults, formed by displacing two halves of a crystal relative to each other along a low-index crystallographic plane, ${ }^{72}$ i.e., the fault plane. As the top half of the crystal moves in the fault plane relative to the bottom half, the crystal's ideal stacking order is disrupted. The resulting energy increase forms the $\gamma$ surface, which is periodic in displacements perpendicular to the faultplane normal. Minima on $\gamma$ surfaces determine possible metastable stacking faults.

We use our EAM potential and DFT to compute sections through the $\{112\}$ and $\{110\} \gamma$ surfaces in the $\langle 111\rangle$ direction. EAM calculations with supercells containing 60000 atoms determine unrelaxed and relaxed $\gamma$-surface energies. The supercell for the $\{112\} \gamma$ surface has 3000 layers and the supercell for the $\{110\} \gamma$ surface has 2000 layers. In each case, the fault plane divides the crystal in half. We calculate the energy as the top half of the crystal is displaced relative to the bottom half along $\langle 111\rangle$. In the relaxed EAM calculations, the atoms are allowed to move only in the direction perpendicular to the fault plane since the stacking faults are unstable. The DFT calculations use supercells with 24 layers for the $\{112\}$ fault plane and 12 layers for the $\{110\}$ fault plane to determine unrelaxed $\gamma$-surface energies.

Figure 6 compares the EAM and DFT $\gamma$-surface sections in the $\langle 111\rangle$ direction for the $\{112\}$ and $\{110\}$ fault planes. There are no minima that would indicate the existence of metastable stacking faults, which is consistent with $\gamma$-surface calculations for many bcc metals. ${ }^{72-77}$ The overall agreement between our EAM and DFT results is very good, but the relaxed (and unrelaxed) EAM results show shoulders near 

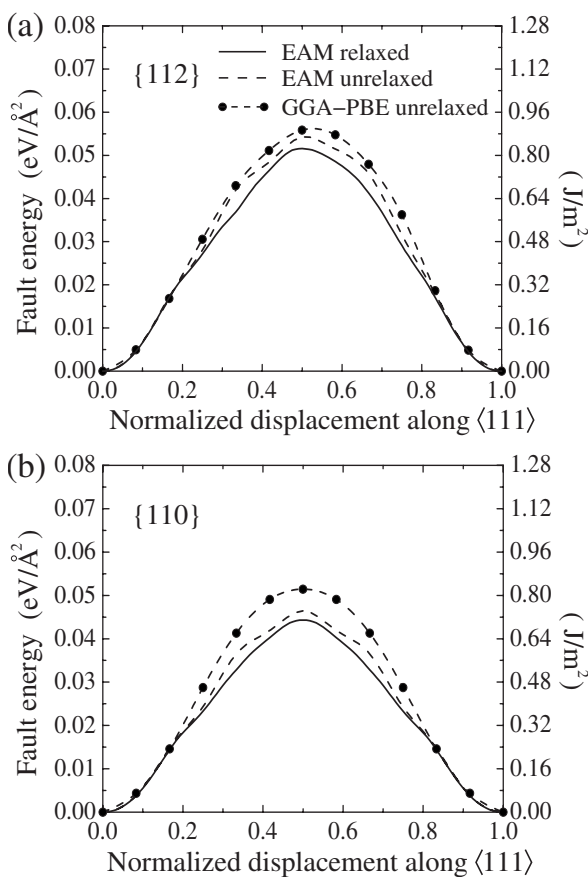

FIG. 6. $\gamma$-surface sections in the $\langle 111\rangle$ direction. The absence of minima indicate that there are no stable stacking faults in the $\{112\}$ and $\{110\}$ planes along this direction. The EAM and DFT results agree well, even though no data from configurations with stacking faults is used to construct the potential.

$b / 6$ and $5 b / 6$ which are absent in the DFT curves, where $b$ is the magnitude of the $(1 / 2)\langle 111\rangle$ screw dislocation Burgers vector $\mathbf{b}=(a / 2)\langle 111\rangle$. Relaxed $\gamma$ surfaces from F-S calculations $^{75}$ show similar shoulders for the group VIB element Mo but not for the group $\mathrm{VB}$ element $\mathrm{Ta}(\mathrm{Nb}$ is also a group VB element).

Figure 7 shows the two types of $(1 / 2)\langle 111\rangle$ screw dislocation core structures found in calculations for bcc metals. Figure 7(a) shows the degenerate core, so named because the configurations on the left and right have the same energy. Figure 7(b) shows the nondegenerate (or symmetric) core. Both types of cores spread into three $\{110\}$ planes of the

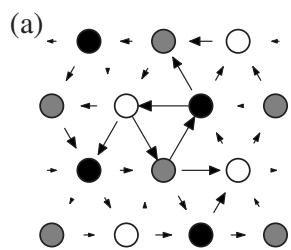

(b)

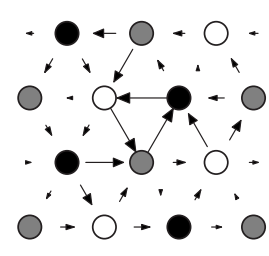

(c)
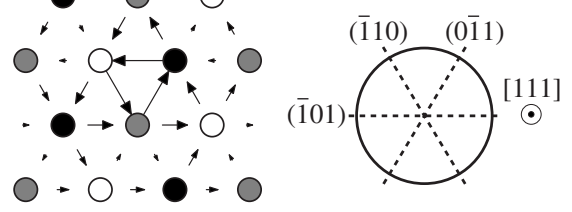

FIG. 7. Differential-displacement maps of the (a) degenerate and (b) nondegenerate (or symmetric) core structures found for (1/ 2)[111] screw dislocations in bcc metals. For the degenerate core, the structures on the left and right have the same energy. (c) In all cases, the core spreads into three $\{110\}$ planes of the [111] zone.

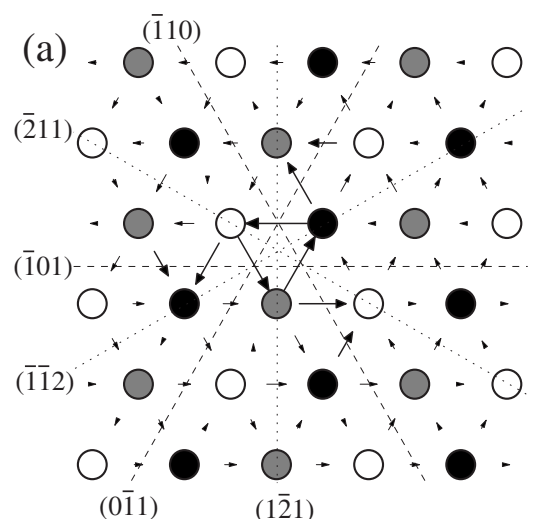

(b)

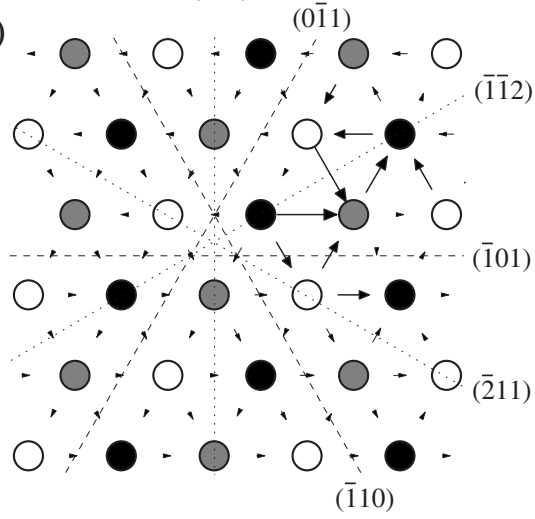

FIG. 8. Differential-displacement maps for the (1/2)[111] screw dislocation core structure produced by our EAM potential. (a) The degenerate core-structure results when the atoms are relaxed. (b) Shear stress applied along [111] produces a net displacement of the dislocation in the $(\overline{1} \overline{1} 2)$ plane. The figure shows the core structure when the shear stress is just above the critical-resolved shear stress for dislocation motion.

[111] zone. The results are presented using differentialdisplacement maps introduced by Vitek et al. ${ }^{78}$ The atoms are projected onto the (111) plane and the arrows represent relative atomic displacements in the [111] direction. The lengths of the arrows are scaled such that an arrow connects two atoms if its length is $b / 3$. The shadings of the atoms indicate that there are three repeating layers of atoms in the [111] direction in an ideal crystal (white is the bottom layer and black is the top layer).

Figure 8(a) shows that our EAM potential for $\mathrm{Nb}$ produces the degenerate core. We determine the core structure for a supercell containing about 900000 atoms. The atoms are arranged in a cylindrical slab oriented such that the $x$ axis is along the $[1 \overline{2} 1]$ direction, the $y$ axis is along [101], and the $z$ axis is along [111]. The radius of the cylinder is $60 \mathrm{~nm}$. The supercell has 15 (111) planes in the $z$ direction. Periodic boundary conditions are applied in the $z$ direction to simulate an infinitely long straight screw dislocation. We insert a (1/ 2)[111] screw dislocation into the ideal crystal by displacing all the atoms in the supercell according to the dislocation's anisotropic elastic strain field. ${ }^{79}$ The resulting structure provides an initial configuration for subsequent conjugategradient relaxation. Atoms that are less than $58 \mathrm{~nm}$ from the center of the cylinder are free to relax (the atomistic region) while the rest of the atoms are fixed at their initial positions. 


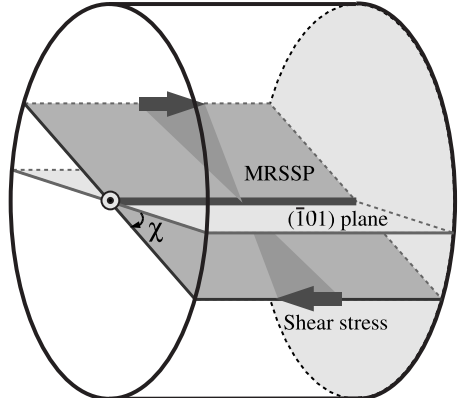

(a)

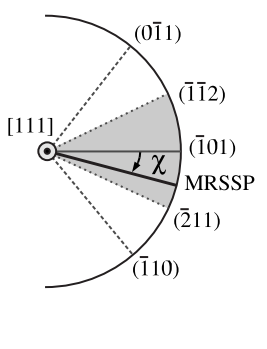

(b)
FIG. 9. Orientation of the maximum resolved shear stress plane (MRSSP) with respect to the ( $\overline{101}$ ) plane. (a) The angle between the MRSSP and the (101) plane is $\chi$. (b) Due to symmetry, we only need to consider $-30^{\circ}<\chi<+30^{\circ}$ (the shaded interval). This range of angles includes the $(\overline{2} 11),(\overline{1} 01)$, and $(\overline{1} 12)$ planes.

This fixed boundary condition effectively extends the dimensions of the system to infinity in the $x$ and $y$ directions. There are no published DFT results for the $(1 / 2)\langle 111\rangle$ core structure in $\mathrm{Nb}$. F-S potentials ${ }^{75}$ produce degenerate cores for the group VIB metals (Cr, Mo, W) and nondegenerate cores for the group VB metals ( $\mathrm{V}, \mathrm{Nb}, \mathrm{Ta})$.

Duesbery and Vitek ${ }^{75}$ propose a criterion that relates the $\{110\} \gamma$ surface to the $(1 / 2)\langle 111\rangle$ screw dislocation core structure. The criterion is based on results from F-S calculations and states that the degenerate core structure forms if

$$
\gamma_{\{110\}}(b / 3)<2 \gamma_{\{110\}}(b / 6),
$$

where $\gamma_{\{110\}}(b / 3)$ and $\gamma_{\{110\}}(b / 6)$ are the $\{110\}$ fault energies at $b / 3$ and $b / 6$ along $\langle 111\rangle$, respectively. Our EAM potential produces $\quad \gamma_{\{110\}}(b / 3)=0.033 \mathrm{eV} / \AA^{2}$ and $\gamma_{\{110\}}(b / 6)$ $=0.014 \mathrm{eV} / \AA^{2}$. These fault energies do not satisfy the criterion for the degenerate core, yet this is the core that our EAM potential favors. This suggests that the Duesbery-Vitek criterion is not generally valid, and that the shapes of the $\gamma$ surfaces and the type of core structure depend on the details of atomic interactions.

Figure 8 shows the relaxed core structure of the (1/2)[111] screw dislocation and its movement under pure shear stress acting parallel to the Burgers vector. We increase the strain on the crystal in small increments and allow the atoms in the atomistic region to relax after each increase in strain. The resulting shear stress acts in the maximum-resolved shear stress plane (MRSSP), and the dislocation moves when the stress reaches the critical-resolved shear stress (CRSS), i.e., the Peierls stress. We compute the CRSS for different orientations of the MRSSP. Figure 9 shows that the orientations of the MRSSP are defined by the angle $\chi$ the MRSSP makes with the (101) plane. It is sufficient to consider $-30^{\circ}<\chi$ $<+30^{\circ}$ due to crystal symmetry. Figure 8(b) shows that when the shear stress reaches the CRSS, the dislocation moves along the $(\overline{1} \overline{1} 2)$ plane for all MRSSP orientations with $\chi<25^{\circ}$. The net motion of the dislocation is in the (1/12) plane. An alternative way to view this motion is the dislocation moves along the $(\overline{101})$ and $(0 \overline{1} 1)$ planes in steps of

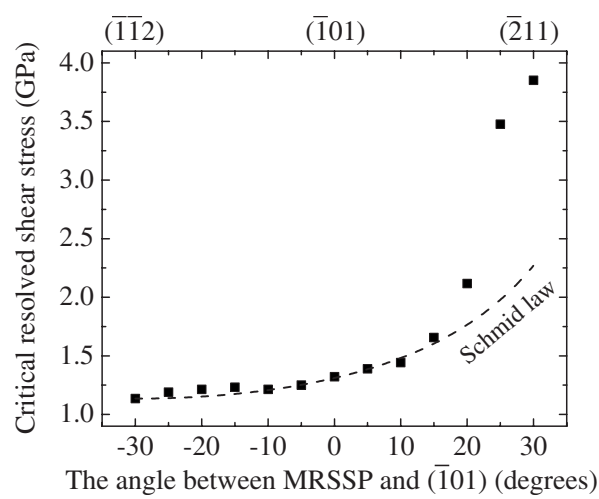

FIG. 10. The critical resolved shear stress (CRSS) for dislocation motion as a function of MRSSP orientation. The figure shows a deviation from the Schmid law when the angle between the MRSSP and the (101) plane is greater than about $15^{\circ}$.

$(1 / 3)[1 \overline{2} 1]$ and $(1 / 3)[\overline{2} 11]$, producing an effective slip in the $(\overline{1} 12)$ plane. The same motion is observed in atomistic simulations of $(1 / 2)[111]$ screw dislocations in Ta using a F-S (Ref. 80) potential and a model generalized pseudopotential theory potential. ${ }^{76}$ Slip on $\{112\}$ and $\{110\}$ planes has been experimentally observed in $\mathrm{Nb}$ single crystals. ${ }^{81-83}$

Figure 10 shows the CRSS for various orientations of the MRSSP. The results clearly demonstrate the dependence of the CRSS on the sense of shearing and illustrates the wellknown breakdown of the Schmid law in bcc metals. ${ }^{64-71}$ This law assumes that components of the stress tensor other than shear in the slip plane in the slip direction play no role in the deformation process and that the critical stress is independent of the sense of shearing. When ( $\overline{1} \overline{1} 2)$ is the slip plane, the Schmid-law dependence of the CRSS on $\chi$ has the form $1 / \cos \left(\chi+30^{\circ}\right)$, drawn as a dashed curve in Fig. 10. Deviations from the Schmid law becomes discernible for $\chi \geqslant 15^{\circ}$, and rapidly increase as the MRSSP approaches the $(\overline{2} 11)$ plane.

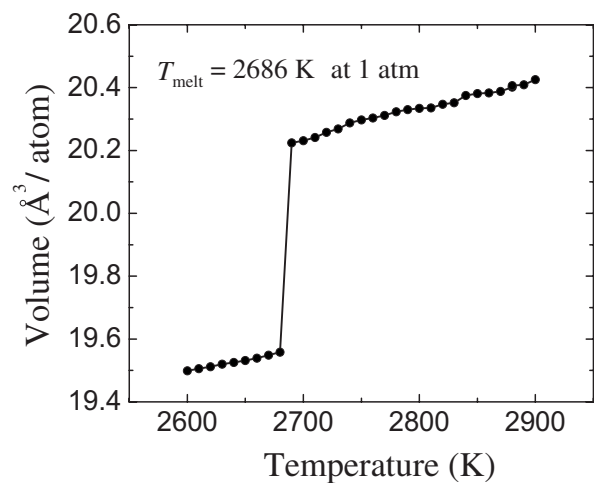

FIG. 11. Two-phase melting simulations determine the melting temperature of our EAM potential. Initially, half the simulation cell contains liquid $\mathrm{Nb}$ and the other half contains bec $\mathrm{Nb}$. The liquid region of the simulation cell solidifies below the melting temperature and the solid region melts above the melting temperature. The figure shows the equilibrium volume for each simulation temperature. There is a sharp jump in volume upon melting. 


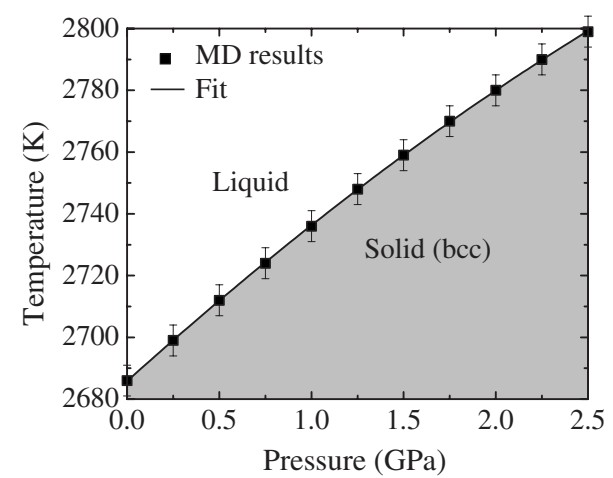

FIG. 12. The melting curve of $\mathrm{Nb}$. The points are EAM results and the solid line fits the values. The melting temperature is computed for pressures to $2.5 \mathrm{GPa}$ with an error of $\pm 5 \mathrm{~K}$ for each temperature. The EAM potential produces a melting temperature of $2685 \mathrm{~K}$ at $P=1 \mathrm{~atm}$. The experimental melting temperature at this pressure is $2742 \mathrm{~K}$. The error between EAM and experiment is only $2 \%$. Melting temperatures have not been measured for higher pressures.

\section{F. Melting}

Our primary interest is solid-state simulations but we also examine melting behavior. Morris et al. ${ }^{84}$ state "for EAM potentials, it has been commonly observed that the melting temperatures are significantly lower $(30 \%$ or more) than experimental values." Accordingly, an accurate melting temperature provides a challenging test for the potential. Twophase melting simulations, in which the simulation cells contain solid and liquid in contact with each other, produce reliable melting temperatures. The liquid-solid interface provides nucleation sites for melting, thereby removing overheating issues associated with single-phase melting simulations. Several methods based on this idea have been applied to the melting of metallic systems. ${ }^{84-89}$

We follow the approach of Belonoshko et al., ${ }^{86}$ in which constant-NPT MD simulations determine the melting temperature. Initially, half the simulation cell is liquid and the other half is bcc. For a given pressure, we compute the average volume for a series of simulations with increasing temperature. The liquid region of the simulation cell solidifies below the melting temperature and the solid region liquefies above the melting temperature. The volume of the system increases sharply across the melting temperature, indicating that a phase transition occurs. The average volume of each phase is constant at the melting point where the two phases coexist. Our simulation cells contain at least 16500 atoms. Each MD simulation runs for 5000000 steps with a 1 fs time step and we average the volumes from the last 5000 steps. We check the coexistence of the phases at the melting temperature using at least five independent simulations. We find that 130000 -atom simulations produce the same melting temperatures as 16 500-atom simulations.

We compute melting temperatures for simulation cells containing liquid in contact with a $\{100\},\{110\}$, or $\{111\}$ bcc surface. Figure 11 shows the increase in volume with temperature at $P=1$ atm for the liquid-\{100\} interface. The melting temperatures at $P=1$ atm from the liquid- $\{100\}$, liquid-
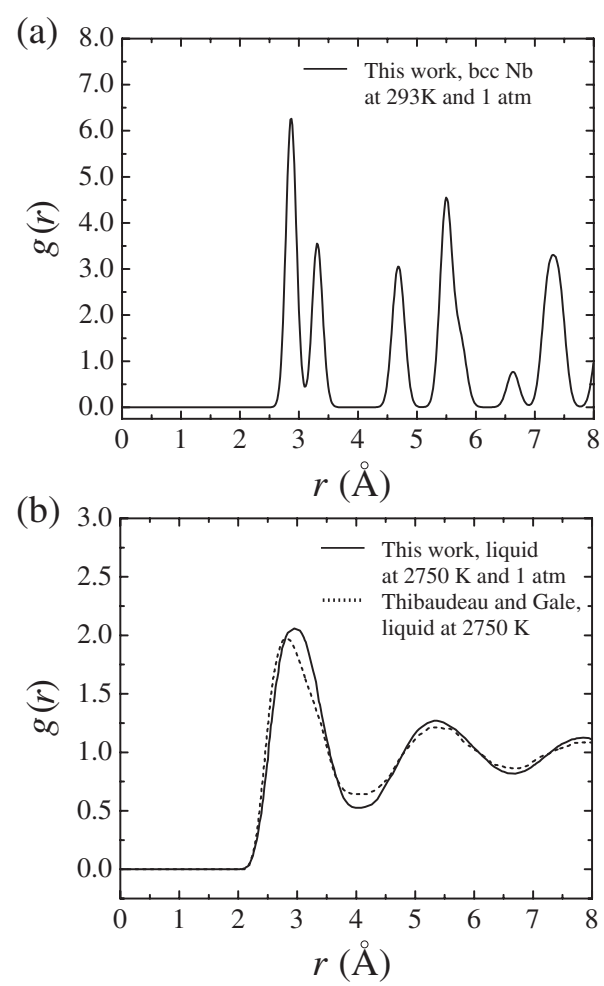

FIG. 13. Radial distribution functions (RDF). (a) $\mathrm{Nb}$ is bcc at $293 \mathrm{~K}$. (b) Liquid $\mathrm{Nb}$ at $2750 \mathrm{~K}$. No experimental data is available, so we compare the liquid result to the RDF from an EAM potential intended for simulating liquid $\mathrm{Nb}$ (Ref. 15). The two potentials produce similar results. The first and second neighbor shells in the solid merge into a single peak in the liquid with similar behavior for higher-order neighbor shells.

$\{110\}$, and liquid-\{111\} simulations are $2686 \mathrm{~K}, 2680 \mathrm{~K}$, and $2688 \mathrm{~K}$, respectively. Each melting temperature has an error of $\pm 5 \mathrm{~K}$. The average of the three melting temperatures is $2685 \mathrm{~K}$. The error between this value and the experimental melting temperature of $2742 \mathrm{~K}$ is only $2 \%$. The agreement is excellent considering that the fitting database does not contain data from configurations near the melting point.

We also determine the melting curve of $\mathrm{Nb}$ for pressures to $2.5 \mathrm{GPa}$. Figure 12 shows the increase in melting temperature with pressure. The points are results from constant-NPT MD simulations, and the solid line is a quadratic fit through the values: $T=T_{0}+\alpha P+\beta P^{2}$, where $T_{0}=2685.8 \pm 0.2 \mathrm{~K}, \alpha$ $=53.9 \pm 0.3 \mathrm{~K} / \mathrm{GPa}$, and $\beta=-3.4 \pm 0.1 \mathrm{~K} / \mathrm{GPa}^{2}$. Each data point has an error of $\pm 5 \mathrm{~K}$. The melting curve of $\mathrm{Nb}$ has not been measured.

Figure 13 shows the radial distribution functions (RDF) for bcc $\mathrm{Nb}$ at $273 \mathrm{~K}$ and $1 \mathrm{~atm}$, and liquid $\mathrm{Nb}$ at $2750 \mathrm{~K}$ and $1 \mathrm{~atm}$. We determine the RDFs by averaging position data from over $1000 \mathrm{MD}$ simulation steps. No experimental data is available for liquid $\mathrm{Nb}$, so we compare our prediction of the liquid RDF to the result from an EAM potential intended for simulating liquid $\mathrm{Nb}{ }^{15}$ Both potentials predict that groups of bcc peaks merge to form wider peaks in the liquid but there are small differences in the maxima of the peaks. 


\section{SUMMARY}

We construct an accurate and reliable EAM potential for $\mathrm{Nb}$ as the first step in alloy potential development. The forcematching program POTFIT optimizes the EAM functions to a database of well-converged DFT forces, energies, and stresses. The potential accurately reproduces properties tied to the fitting data and shows excellent agreement with DFT and experiment for a large number of other quantities that are related to configurations not included in the fitting database. The potential describes structural and elastic properties, defects, and thermodynamic behavior. While the potential may not be well suited for shock-wave or radiation damage studies, it performs very well in all other situations we have tested. The potential also serves as a viable starting point for constructing accurate EAM potentials for $\mathrm{Nb}$ alloys.

\section{ACKNOWLEDGMENTS}

We thank Richard G. Hennig for providing the DFT phonon results. We thank Richard G. Hennig, Thomas J. Lenosky, Dallas R. Trinkle, and Murray S. Daw for useful discussions. This work was supported by DOE-Basic Energy Sciences, Division of Materials Sciences (Grant No. DEFG02-99ER45795). Computational resources were provided in part by an allocation of computing time from the Ohio Supercomputer Center. This research also used resources of the National Energy Research Scientific Computing Center, which is supported by the Office of Science of the U.S. Department of Energy under Contract No. DE-AC0205CH11231.

\section{APPENDIX: FUNCTION MODIFICATIONS}

In this appendix we discuss modifications to $\phi(r)$ and $\rho(r)$ for small $r$, and to $F(n)$ for small and large $n$. In MD simulations, fluctuations can move atoms closer together than the minimum interatomic distance in the fitting database. The POTFIT program accounts for this by extending $\phi$ and $\rho$ to $r$ values smaller than the inner cutoff radius. The cubic polynomials in the range $2.073<r<2.240 \AA$ are extended down to $1.738<r<2.240 \AA$ A. Likewise, POTFIT extends $F$ to $n$ values smaller than the inner cutoff, and $n$ values larger than the outer cutoff. The cubic polynomial in the range $0.0775<n$ $<0.209$ is extended down to $-0.0186<n<0.209$, and a very steep cubic polynomial is added for $1.000<n<1.264$. Requiring continuity of $F$ and its first and second derivatives at $n=1.000$ determines three coefficients of the steep cubic function. Setting $F$ equal to $4.828 \mathrm{eV}$ at $n=1.264$ determines the final coefficient.

Despite these modifications, the potential is not repulsive enough for high-temperature and high-pressure simulations, where atoms closely approach one another. To overcome this limitation, we modify $\phi$ for $1.738<r<2.073 \AA$. We replace the function in this range by a steeper cubic polynomial. The continuity of $\phi$ and its first and second derivatives at $r$ $=2.073 \AA$ determines three coefficients. Setting the first derivative at $r=1.738 \AA$ equal to four times the first derivative at $r=2.073 \AA$ determines the final coefficient. This modified potential is repulsive enough at small atomic separations to prevent collapse problems for all temperature and pressure ranges we investigated.

We also modify the extension of $F$ for small $n$ values to properly describe the cohesive energy. The minimum of the EAM energy per atom versus volume curve for bcc $\mathrm{Nb}$ equals the cohesive energy but the embedding energy is not zero for $n=0$ when the atoms are far apart. Therefore, we replace the POTFIT modification for small $n$ by a different cubic polynomial. We choose three coefficients to ensure continuity of the embedding function and its first and second derivatives at $n=0.0775$. We determine the final coefficient by setting $F(0)=0$. Table II lists the optimized spline knots, boundary conditions, and modifications of $\phi, \rho$, and $F$.
*Corresponding author; mfelling@mps.ohio-state.edu

${ }^{1}$ T. Saito, T. Furuta, J.-H. Hwang, S. Kuramoto, K. Nishino, N. Suzuki, R. Chen, A. Yamada, K. Ito, Y. Seno, T. Nonaka, H. Ikehata, N. Nagasako, C. Iwamoto, T. Ikuhara, and T. Sakuma, Science 300, 464 (2003).

${ }^{2}$ G. Ghosh and G. B. Olson, Acta Mater. 55, 3281 (2007).

${ }^{3}$ J. I. Kim, H. Y. Kim, H. Hosoda, and S. Miyazaki, Mater. Trans. 46, 852 (2005).

${ }^{4}$ M. W. Finnis and J. E. Sinclair, Philos. Mag. A 50, 45 (1984).

${ }^{5}$ G. J. Ackland and R. Thetford, Philos. Mag. A 56, 15 (1987).

${ }^{6}$ R. Rebonato, D. O. Welch, R. D. Hatcher, and J. C. Bilello, Philos. Mag. A 55, 655 (1987).

${ }^{7}$ R. A. Johnson and D. J. Oh, J. Mater. Res. 4, 1195 (1989).

${ }^{8}$ A. M. Guellil and J. B. Adams, J. Mater. Res. 7, 639 (1992).

${ }^{9}$ M. I. Baskes, Phys. Rev. B 46, 2727 (1992).

${ }^{10}$ Z. Bangwei, Y. Ouyang, S. Liao, and Z. Jin, Physica B 262, 218 (1999).

${ }^{11}$ B.-J. Lee, M. I. Baskes, H. Kim, and Y. K. Cho, Phys. Rev. B 64, 184102 (2001).
${ }^{12}$ W. Hu, X. Shu, and B. Zhang, Comput. Mater. Sci. 23, 175 (2002).

${ }^{13}$ X. D. Dai, J. H. Li, and Y. Kong, Phys. Rev. B 75, 052102 (2007).

${ }^{14}$ Y.-N. Wen and J.-M. Zhang, Comput. Mater. Sci. 42, 281 (2008).

${ }^{15}$ P. Thibaudeau and J. Gale, arXiv:0809.0198 (unpublished).

${ }^{16}$ F. Ercolessi and J. B. Adams, Europhys. Lett. 26, 583 (1994).

${ }^{17}$ P. Hohenberg and W. Kohn, Phys. Rev. 136, B864 (1964).

${ }^{18}$ W. Kohn and L. J. Sham, Phys. Rev. 140, A1133 (1965).

${ }^{19}$ M. S. Daw and M. I. Baskes, Phys. Rev. Lett. 50, 1285 (1983).

${ }^{20}$ M. S. Daw and M. I. Baskes, Phys. Rev. B 29, 6443 (1984).

${ }^{21}$ P. Brommer and F. Gähler, Philos. Mag. 86, 753 (2006).

${ }^{22}$ P. Brommer and F. Gähler, Modell. Simul. Mater. Sci. Eng. 15, 295 (2007); more information about POTFIT is found at http:// www.itap.physik.uni-stuttgart.de/ imd/potfit

${ }^{23}$ A. E. Carlsson, in Solid State Physics, edited by H. Ehrenreich and D. Turnbull (Academic, New York, 1990), Vol. 43, pp. 1-91.

${ }^{24}$ R. A. Johnson, Phys. Rev. B 6, 2094 (1972). 
${ }^{25}$ M. J. Stott and E. Zaremba, Phys. Rev. B 22, 1564 (1980).

${ }^{26}$ J. K. Nørskov, Phys. Rev. B 26, 2875 (1982).

${ }^{27}$ J. Stadler, R. Mikulla, and H.-R. Trebin, Int. J. Mod. Phys. C 8, 1131 (1997)

${ }^{28}$ J. Roth, F. Gähler, and H.-R. Trebin, Int. J. Mod. Phys. C 11, 317 (2000); more information about IMD is found at http:// www.itap.physik.uni-stuttgart.de/ imd/

${ }^{29}$ S. J. Plimpton, J. Comput. Phys. 117, 1 (1995); more information about LAMMPS is found at http://lammps.sandia.gov

${ }^{30}$ J. Kim, OHMMS, http://mcc1.mcc.uiuc.edu/ ohmms/, 2004

${ }^{31}$ G. Kresse and J. Furthmüller, Phys. Rev. B 54, 11169 (1996).

${ }^{32}$ J. P. Perdew, K. Burke, and M. Ernzerhof, Phys. Rev. Lett. 77, 3865 (1996).

${ }^{33}$ P. E. Blöchl, Phys. Rev. B 50, 17953 (1994).

${ }^{34}$ G. Kresse and D. Joubert, Phys. Rev. B 59, 1758 (1999).

${ }^{35}$ M. Methfessel and A. T. Paxton, Phys. Rev. B 40, 3616 (1989).

${ }^{36}$ I. J. Robertson, V. Heine, and M. C. Payne, Phys. Rev. Lett. 70, 1944 (1993).

${ }^{37}$ Y. Mishin, D. Farkas, M. J. Mehl, and D. A. Papaconstantopoulos, Phys. Rev. B 59, 3393 (1999).

${ }^{38}$ Y. Li, D. J. Siegel, J. B. Adams, and X.-Y. Liu, Phys. Rev. B 67, 125101 (2003).

${ }^{39}$ R. G. Hennig, T. J. Lenosky, D. R. Trinkle, S. P. Rudin, and J. W. Wilkins, Phys. Rev. B 78, 054121 (2008).

${ }^{40}$ F. Birch, J. Geophys. Res. 83, 1257 (1978).

${ }^{41}$ M. J. Mehl, B. M. Klein, and D. A. Papaconstantopoulos, in Intermetallic Compounds: Principles and Practice, edited by J. H. Westbrook and R. L. Fleischer (Wiley, London, 1995), Vol. I, pp. 195-210.

${ }^{42}$ The third-order Birch-Murnaghan equation of state is $E_{\mathrm{BM}}(V)$ $=E_{0}+(9 / 8) B V_{0}\left[\left(V_{0} / V\right)^{2 / 3}-1\right]^{2}+(9 / 16) B V_{0}\left(B^{\prime}-4\right)\left[\left(V_{0} / V\right)^{2 / 3}\right.$

$-1]^{3}$, where $E_{0}$ is the minimum of the energy vs volume curve, $B$ is the bulk modulus, $V_{0}$ is the equilibrium volume, and $B^{\prime}$ is the pressure derivative of $B$.

${ }^{43}$ C. Kittel, Introduction to Solid State Physics, 7th ed. (Wiley, New York, 1996).

${ }^{44}$ R. Roberge, J. Less-Common Met. 40, 161 (1975).

${ }^{45}$ G. Simmons and H. Wang, Single Crystal Elastic Constants and Calculated Aggregate Properties: A Handbook, 2nd ed. (The MIT Press, Cambridge, 1971).

${ }^{46}$ G. Mills and H. Jónsson, Phys. Rev. Lett. 72, 1124 (1994).

${ }^{47}$ G. Mills, H. Jónsson, and G. K. Schenter, Surf. Sci. 324, 305 (1995).

${ }^{48}$ H. Jónsson, G. Mills, and K. W. Jacobson, in Classical and Quantum Dynamics in Condensed Phase Simulations, edited by B. J. Berne, G. Ciccotti, and D. F. Coker (World Scientific, Singapore, 1998), pp. 385-404.

${ }^{49}$ E. Polak and G. Ribière, Rev. Fr. Inform. Rech. Oper. 16, 35 (1969).

${ }^{50}$ W. H. Press, S. A. Teukolsky, W. T. Vetterling, and B. P. Flannery, Numerical Recipes: The Art of Scientific Computing, 3rd ed. (Cambridge University Press, New York, 2007).

${ }^{51}$ J. M. Harder and D. J. Bacon, Philos. Mag. A 54, 651 (1986).

${ }^{52}$ Atomic Defects in Metals, Landolt-Börnstein, New Series, Group III Vol. 25, edited by H. Ullmaier (Springer-Verlag, Berlin, 1991).

${ }^{53}$ D. Ablitzer, Philos. Mag. 35, 1239 (1977).

${ }^{54}$ R. E. Einziger, J. N. Mundy, and H. A. Hoff, Phys. Rev. B 17, 440 (1978)

${ }^{55}$ W. Bussmann, C. Herzig, H. A. Hoff, and J. N. Mundy, Phys.
Rev. B 23, 6216 (1981).

${ }^{56} \mathrm{R}$. W. Siegel, in Proceedings of the Fifth Yamada Conference on Point Defects and Defect Interactions in Metals, edited by J.-I. Takamura, M. Doyama, and M. Kiritani (University of Tokyo Press, Tokyo, 1982), pp. 533-540.

${ }^{57}$ J. M. Harder and D. J. Bacon, Philos. Mag. A 58, 165 (1988).

${ }^{58}$ D. Alfè, Comput. Phys. Commun. 180, 2622 (2009).

${ }^{59}$ B. M. Powell, P. Martel, and A. D. B. Woods, Phys. Rev. 171, 727 (1968).

${ }^{60}$ Y. S. Touloukian, R. K. Kirby, R. E. Taylor, and P. D. Desai, Thermophysical Properties of Matter: Thermal ExpansionMetallic Elements and Alloys (Plenum Press, New York, 1975), Vol. 12.

${ }^{61}$ R. Kinslow, High-Velocity Impact Phenomena (Academic Press, New York, 1970).

${ }^{62}$ G. J. Ackland and M. W. Finnis, Philos. Mag. A 54, 301 (1986).

${ }^{63}$ W.-S. Lo, T.-S. Chien, B.-S. Fang, C. M. Wei, and W. N. Mei, Surf. Rev. Lett. 5, 1035 (1998).

${ }^{64}$ L. P. Kubin, Rev. Deform. Behav. Mater. 4, 181 (1982).

${ }^{65}$ J. W. Christian, Metall. Trans. A 14, 1237 (1983).

${ }^{66}$ M. S. Duesbery, in Dislocations in Solids, edited by F. R. N. Nabarro (Elsevier, Amsterdam, 1989), Vol. 8, pp. 66-173.

${ }^{67}$ V. Vitek, Prog. Mater. Sci. 36, 1 (1992).

${ }^{68}$ A. Seeger, J. Phys. IV 05, C7-45-C7-65 (1995).

${ }^{69}$ W. Pichl, Phys. Status Solidi A 189, 5 (2002).

${ }^{70}$ M. S. Duesbery, V. Vitek, and J. Cserti, in Understanding Materials, edited by C. J. Humphreys (Maney, London, 2002), pp. 165-192.

${ }^{71}$ R. Gröger and V. Vitek, Philos. Mag. Lett. 87, 113 (2007).

${ }^{72}$ V. Vítek, Philos. Mag. A 18, 773 (1968).

${ }^{73}$ N. I. Medvedeva, O. N. Mryasov, Y. N. Gornostyrev, D. L. Novikov, and A. J. Freeman, Phys. Rev. B 54, 13506 (1996).

${ }^{74}$ W. Xu and J. A. Moriarty, Phys. Rev. B 54, 6941 (1996).

${ }^{75}$ M. S. Duesbery and V. Vitek, Acta Mater. 46, 1481 (1998).

${ }^{76}$ L. H. Yang, P. Soderlind, and J. A. Moriarty, Philos. Mag. A 81, 1355 (2001).

${ }^{77}$ S. L. Frederiksen and K. W. Jacobsen, Philos. Mag. 83, 365 (2003).

${ }^{78}$ V. Vitek, R. C. Perrin, and D. K. Bowen, Philos. Mag. 21, 1049 (1970).

${ }^{79}$ J. P. Hirth and J. Lothe, Theory of Dislocations, 2nd ed. (Krieger, Malabar, 1982).

${ }^{80}$ K. Ito and V. Vitek, Philos. Mag. A 81, 1387 (2001).

${ }^{81}$ M. S. Duesbery, R. A. Foxall, and P. B. Hirsch, J. Phys. (Paris) 27, C3-193-C3-204 (1966)

${ }^{82}$ R. A. Foxall, M. S. Duesbery, and P. B. Hirsch, Can. J. Phys. 45, 607 (1967)

${ }^{83}$ J.-Y. Kim, D. Jang, and J. R. Greer, Scr. Mater. 61, 300 (2009).

${ }^{84}$ J. R. Morris, U. Dahlborg, and M. Calco-Dahlborg, J. Non-Cryst. Solids 353, 3444 (2007).

${ }^{85}$ J. R. Morris, C. Z. Wang, K. M. Ho, and C. T. Chan, Phys. Rev. B 49, 3109 (1994).

${ }^{86}$ A. B. Belonoshko, R. Ahuja, and B. Johansson, Phys. Rev. Lett. 84, 3638 (2000).

${ }^{87}$ A. Laio, S. Bernard, G. L. Chiarotti, S. Scandolo, and E. Tosatti, Science 287, 1027 (2000).

${ }^{88}$ D. Alfè, M. J. Gillan, and G. D. Price, J. Chem. Phys. 116, 6170 (2002).

${ }^{89}$ J. R. Morris and X. Song, J. Chem. Phys. 116, 9352 (2002). 